\title{
Evaluation of a phenomenological elastic-plastic approach for magnesium alloys under multiaxial loading conditions
}

\author{
Vitor Anes ${ }^{1}$ (D) | Luís Reis ${ }^{2}$ (D) | Manuel de Freitas ${ }^{2}$
}

\author{
${ }^{1}$ Instituto Superior de Engenharia de \\ Lisboa, Instituto Politécnico de Lisboa, \\ 1959-007 Lisbon, Portugal \\ ${ }^{2}$ IDMEC, Instituto Superior Técnico, \\ Universidade de Lisboa, 1049-001 Lisbon, \\ Portugal

\section{Correspondence} \\ Vitor Anes, Instituto Superior de \\ Engenharia de Lisboa, Instituto \\ Politécnico de Lisboa, Rua Conselheiro \\ Emídio Navarro, 1, 1959-007 Lisbon, \\ Portugal. \\ Email: vanes@dem.isel.pt

\section{Funding information} \\ Fundação para a Ciência e a Tecnologia, \\ Grant/Award Number: Ph.D. Grant \\ PD/BD/52344/2013; Fundação para \\ Ciência e Tecnologia project PTDC/EME- \\ PME/104404/2008
}

\begin{abstract}
Magnesium alloys are greatly appreciated due to their high strength to weight ratio, stiffness, and low density; however, they can exhibit complex types of cyclic plasticity like twinning, de-twinning, or Bauschinger effect. Recent studies indicate that these types of cyclic plastic deformations cannot be fully characterized using the typical tools used in cyclic characterization of steels and aluminium alloys; thus, it is required new approaches to fully capture their cyclic deformation and plasticity. This study aims to propose and evaluate a phenomenological cyclic elastic-plastic approach designed to capture the cyclic deformation of magnesium alloys under multiaxial loading conditions. Series of experimental tests were performed to characterize the cyclic mechanical behaviour of the magnesium alloy AZ31BF considering proportional loadings with different strain amplitude ratios and a nonproportional loading with a $45^{\circ}$ phase shift. The experimental results were modulated using polynomial functions in order to implement a cyclic plasticity model for the AZ311BF based on the phenomenological approach proposed. Results show good correlations between experiments and estimates.
\end{abstract}

\section{KEYWORDS}

AZ31B-F, cyclic plasticity, experimental tests, magnesium alloy, multiaxial loading paths

\section{1 | INTRODUCTION}

Nowadays, industry in general is following with great attention the magnesium alloys' research. The positive results achieved by the scientific community have created expectations to improve existing products and create new ones. One example is the automotive industry which from the 50s, until now, has been one of the biggest players in this research field. Despite the improvements in fuel consumption and vehicle performance be an economic approach and a driving force from the customer side, the reduction of fuel consumption is also motivated by legislative rules to reduce primary energy consumption, and environmental impacts; thus, it starts to be a mandatory requirement. The combination of high strength and low weight remains a paramount goal that

Nomenclature: $\lambda$, Strain amplitude ratio; $\varepsilon_{s l}$, Strain level; $\gamma$, Shear strain; $\varepsilon$, Axial strain; $\gamma_{t}$, Total shear strain; $\varepsilon_{t}$, Total axial strain; $a_{\varepsilon_{t}}, \mathrm{~b}_{\varepsilon_{t}}, \mathrm{c}_{\varepsilon_{t}}, \mathrm{~d}_{\varepsilon_{t}}$, Polynomial constants_right branch of axial stress hysteresis loop; $e_{\varepsilon_{t}}, \mathrm{f}_{\varepsilon_{t}}, \mathrm{~g}_{\varepsilon_{t}}, \mathrm{~h}_{\varepsilon_{t}}$, Polynomial constants-left branch of axial stress hysteresis loop; $a_{\gamma_{t}}, \mathrm{~b}_{\gamma_{t}}, \mathrm{c}_{\gamma_{t}}, \mathrm{~d}_{\gamma_{t}}$, Polynomial constants-right branch of shear stress hysteresis loop; $e_{\gamma_{t}}, \mathrm{f}_{\gamma_{t}}, \mathrm{~g}_{\gamma_{t}}, \mathrm{~h}_{\gamma_{t}}$, Polynomial constants-left branch of shear stress hysteresis loop; $a_{i}, b_{i}, c_{i}, d_{i}, e_{i}, f_{i}, g_{i}, h_{i}, i_{i}, j_{i}$, Polynomial constants for axial P functions; $a_{j}, b_{j}, c_{j}, d_{j}, e_{j}, f_{j}, g_{j}, h_{j}, i_{j}, j_{j}$, Polynomial constants for shear P functions; $P_{\text {axial }, i}\left(\varepsilon_{s l}, \lambda\right)$, Axial P function for point $i$; $P_{\text {shear }, j}\left(\varepsilon_{s l}, \lambda\right)$, Shear P function for point $j$

Abbreviations: SAR, Strain amplitude ratio, stress amplitude ratio; H, Phenomenological model; exp, Experimental results 
continues under pursuit by mechanical engineers in order to fulfil the transportation industry requirements. Until now, magnesium alloys are the structural materials that better fulfil this goal. The weight reduction requires a compromise between the material properties and geometry of structural components; under this equilibrium, a full understanding of cyclic, monotonic, and elasticplastic properties is needed in all loading regimens.

Cyclic plasticity is quite different from plasticity under static loadings. Materials when subjected to cyclic loads tend to modify their mechanical properties, and due to that, stress states of mechanical components can be quite different from the ones considered in design stages if cyclic plasticity is not taken into account. ${ }^{1}$

Mechanical components and structures are generally subjected to multiaxial loadings with variable amplitude; therefore, multiaxial cyclic plasticity models are needed in their design. ${ }^{2}$ However, constitutive plasticity models ${ }^{3,4}$ of commercial finite element packages have as input uniaxial cyclic curves to set up their internal plasticity routines; this means that the yield functions, hardening, and flow rules commonly used in these packages are not suitable to simulate multiaxial cyclic plasticity, which depends on many factors such as the stress ratio between normal and shear stresses or nonproportional effects. This unsuitability increases for materials with nonstandard cyclic behaviour, specially magnesium alloys.

Albinmusa $^{2,5,6}$ reinforced this idea and stated that it is needed anisotropic plasticity models to cover the cyclic behaviour of magnesium alloys. It is impossible to estimate this anisotropy using only the uniaxial stress-strain relation as found in constitutive models. From experiments, it was found out that magnesium alloys have a nonlinear cyclic behaviour, which is dependent on many factors such as strain rate and microstructure deformation mechanisms. ${ }^{1}$ Therefore, instead of using the uniaxial stress-strain relation under cyclic loadings as usually is, it is necessary to use several different types of cyclic loadings to map the material cyclic anisotropy.

There are very few works in literature that focus the multiaxial stress-strain relations for magnesium alloys, ${ }^{6-}$ ${ }^{11}$ but all of them stated that the magnesium alloys cyclic behaviour is quite different from steels or even from aluminium alloys. One evidence of this fact can be seen in the typical asymmetric hysteresis loops found in magnesium alloys, where the cyclic yield stress at compression is quite different from the one in tension; also, the rate variation of these values is also different. ${ }^{12,13}$

Commercial finite element packages are not able to modulate the anisotropy found in magnesium alloys; they consider that ductile materials have the same yield stress in tension and compression for a given strain level, which is true for steels and aluminium alloy but not true for magnesium alloys. ${ }^{2,7}$ Moreover, back stresses in tension and compression have also different absolute values in magnesium alloys for a given strain level. ${ }^{13}$ The complexity increases when it is considered in multiaxial loading conditions, where yield stresses, hardening, and flow rules vary differently according to the loading type and load level. ${ }^{14}$

Moreover, in literature, it can be found studies indicating that equivalent stress/strains approaches do not have into account important effects such as the material strength dependence on the loading direction; therefore, it is possible to obtain the same equivalent stress in all loading directions. Nevertheless, the cyclic response of magnesium alloys is different according to the loading direction. ${ }^{15}$ In this sense, equivalent stress/strains used in hardening rules and flow rules must capture the material anisotropy to estimate their cyclic behaviour under multiaxial loading conditions. One way to do that is to adopt a phenomenological approach and perform specific experimental tests under multiaxial loading conditions to obtain a map of the material cyclic response in order to create or to improve these rules. ${ }^{14,16}$

There are three types of cyclic plasticity models, namely: constitutive, phenomenological, and constitutive-phenomenological models. Constitutive models are based on the solid mechanics' approach, and they use very few experimental data, ie, typical mechanical properties are used to estimate the material cycle response. ${ }^{17}$ This approach is very appreciated; however, it has strong limitations in magnesium alloys and anisotropic materials. On the other hand, phenomenological plasticity models are mainly based on experiments. These models should cover the basic premises of constitutive models, ie, must capture an experimental yield function, hardening, and flow rules. Both approaches try to estimate the same cyclic behaviour but following different ways. Constitutive models cover a wide range of materials and do not need special experimental programs, but for anisotropic materials they a have limited performance. Therefore, it is advised phenomenological cyclic models to model the cyclic behaviour of anisotropic materials including magnesium alloys. The constitutivephenomenological models make use of both approaches, usually developed to integrate the information given by phenomenological models into constitutive models.

This work presents a phenomenological elastic-plastic approach to implement phenomenological models for anisotropic materials. This approach is based on experimental stress-strain relations under a wide range of cyclic strains under uniaxial and specific multiaxial loading conditions. The proposed approach is applied to the magnesium alloy AZ31BF in order to implement its phenomenological elastic-plastic cyclic model. 
$1 \& 4$ Total strain

$2 \& 5$ Plastic strain

$3 \& 6$ Back stress
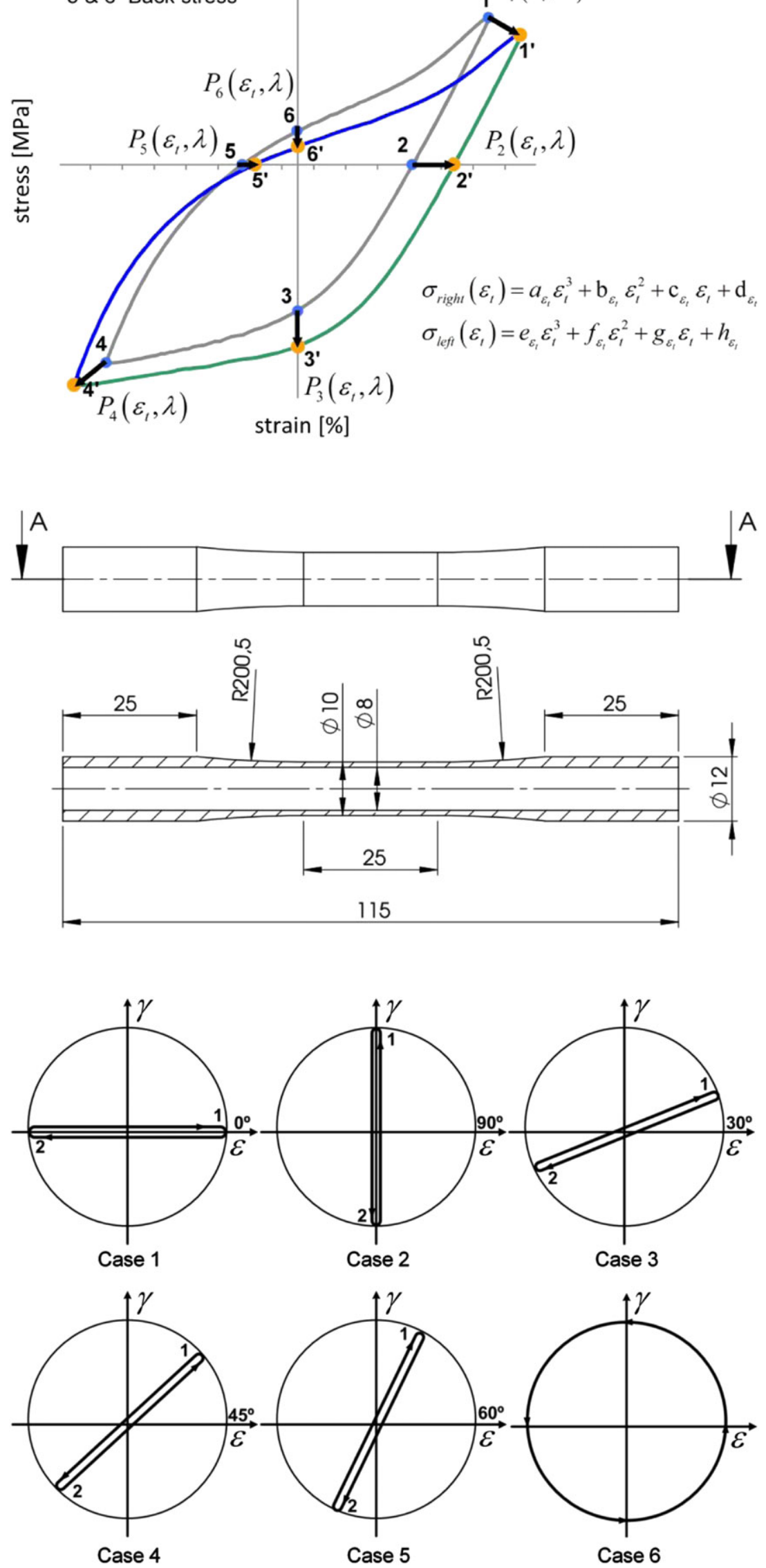

FIGURE 1 Magnesium alloy hysteresis loop typically obtained from the axial channel of a biaxial loading [Colour figure can be viewed at wileyonlinelibrary.com]

FIGURE 2 Specimen test geometry and dimensions

FIGURE 3 Loading paths tested in strain control: A, Case 1, PT; B, Case 2, PS; C, Case 3, PP30; D, Case 4, PP45; E, Case 5, PP60; and, F, Case 6, OP45 


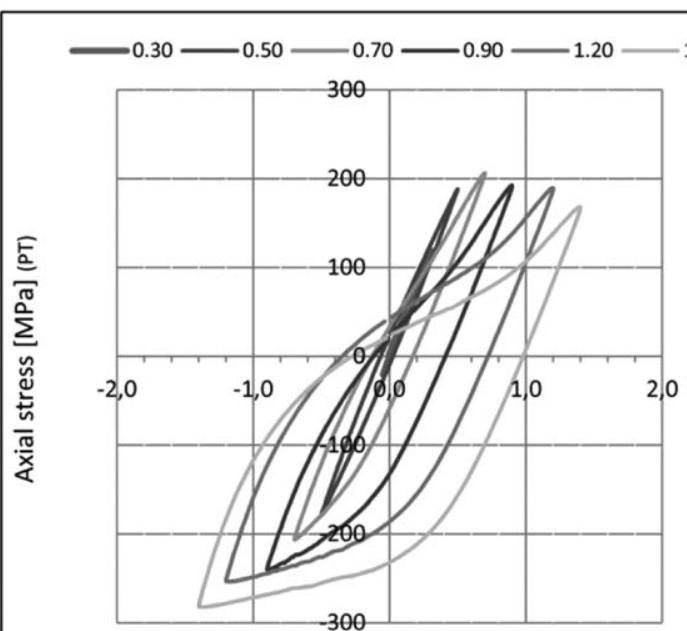

(A)

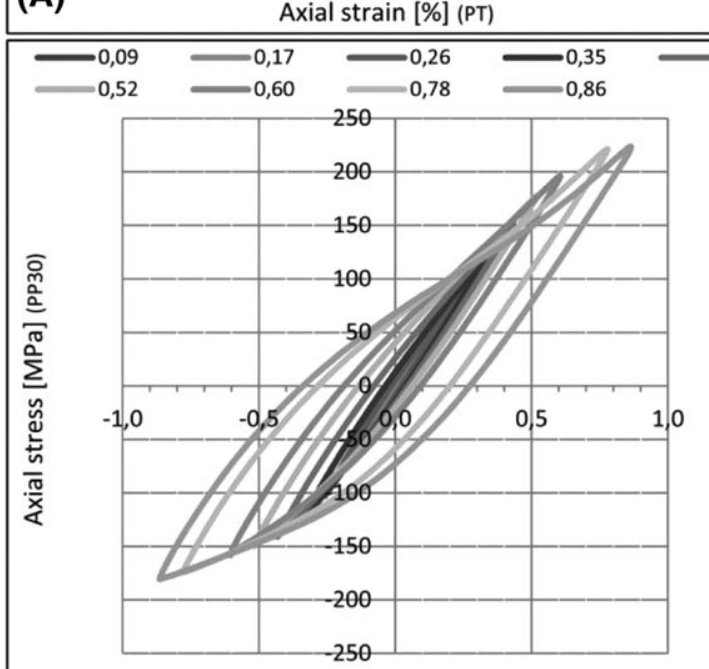

(C)

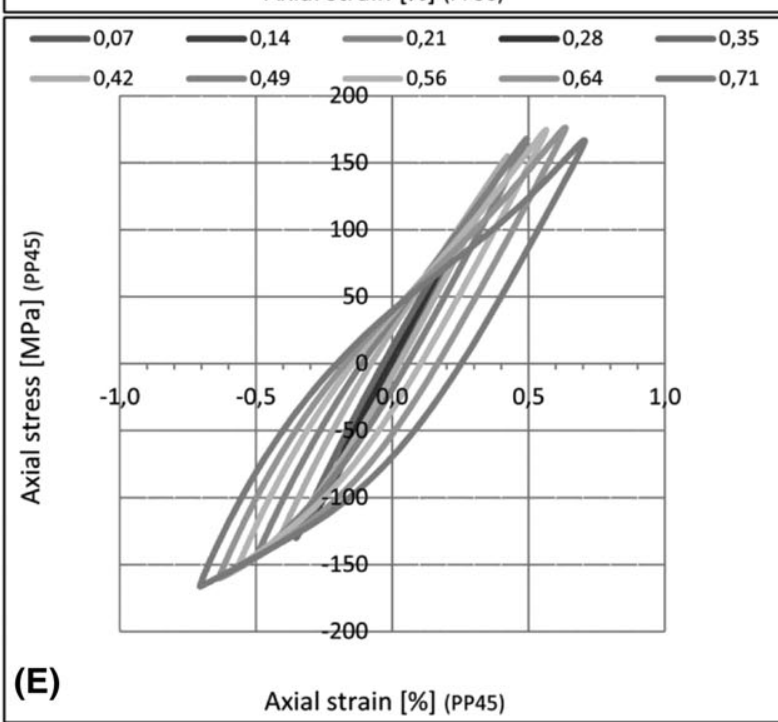

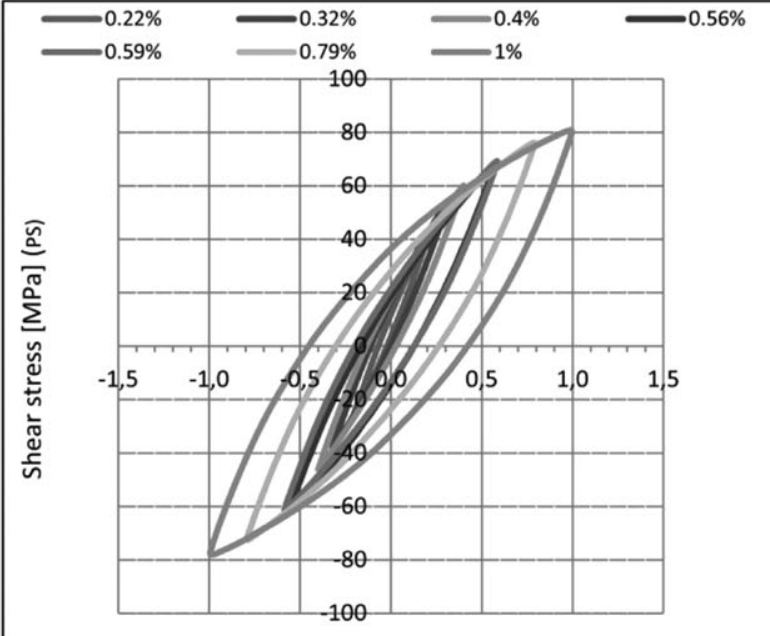

(B)

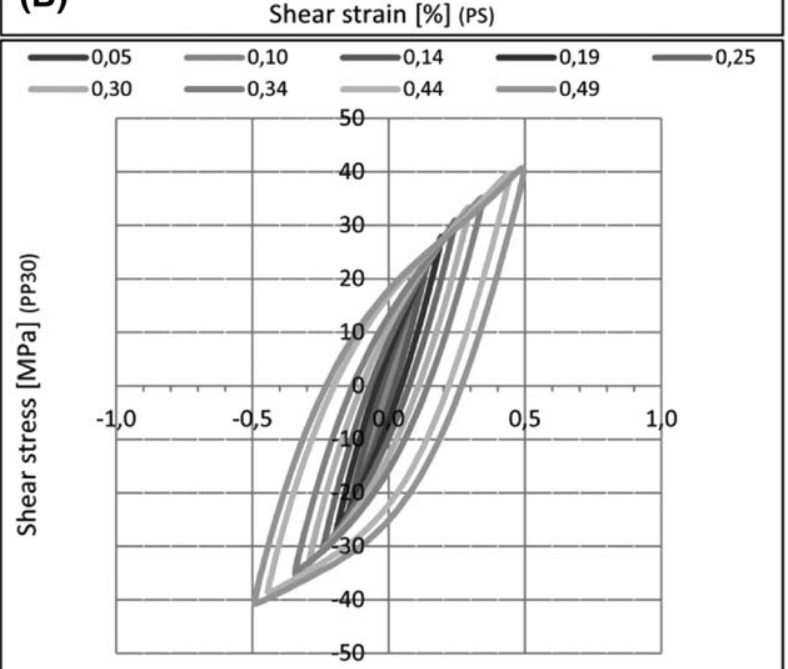

(D) Shear strain [\%] (PP30)

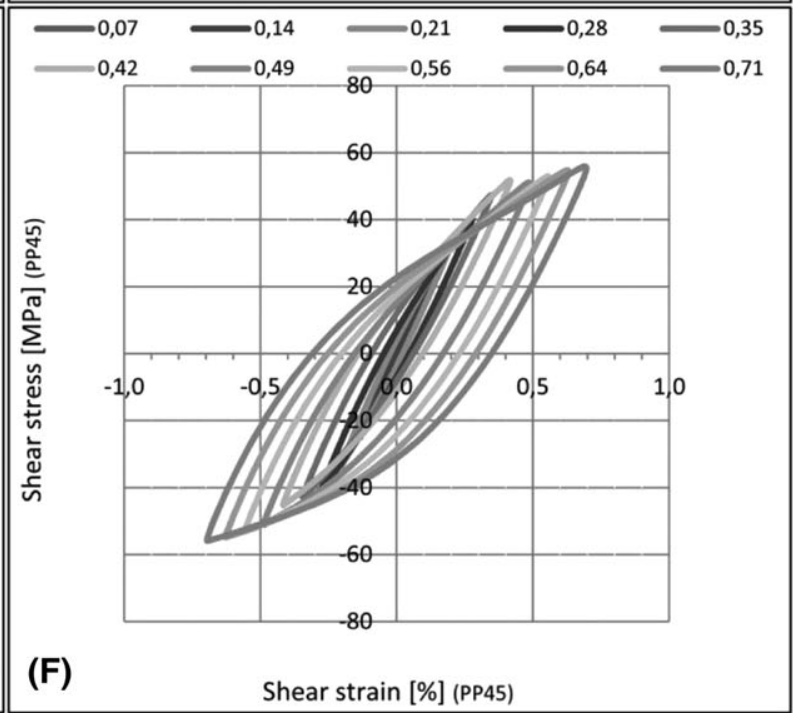

FIGURE 4 Experimental hysteresis loops for A, Case 1; B, Case 2; C, Case 3-axial component; D, Case 3-shear component; E, Case 4Axial component; F, Case 4-shear component 


\section{2 | PHENOMENOLOGICAL ELASTIC-PLASTIC APPROACH}

The cyclic mapping of magnesium alloys is a feasible way to overcome the limitations found in constitutive cyclic plasticity models. A phenomenological approach like the one presented in Section 2.1 allows to implement models that mimic the cyclic response of anisotropic materials which can be implemented in an external routine linked to a finite element package instead of using internal constitutive models offered by these packages. In this way, the material cyclic behaviour can be cyclic updated with this external routine according to the cyclic loading features.

The objective of the proposed phenomenological elastic-plastic approach is to set several steps to achieve a numeric model able to estimate the relation between stresses and strains in uniaxial and multiaxial loading conditions for a total strain range with practical applications in mechanical design.

\section{1 | Phenomenological approach}

From experiments, it was found that the AZ31 magnesium alloy hysteresis loops can be approximated with very acceptable results using a third-degree polynomial function for any value of total strain.

In order to obtain these functions, it is considered six specific points on a hysteresis loop, see Figure 1. For the left hysteresis branch, the polynomial function is obtained by interpolation using the experimental data obtained at points $4,5,6$, and 1 . Similarly, the polynomial function of the hysteresis branch at right is obtained

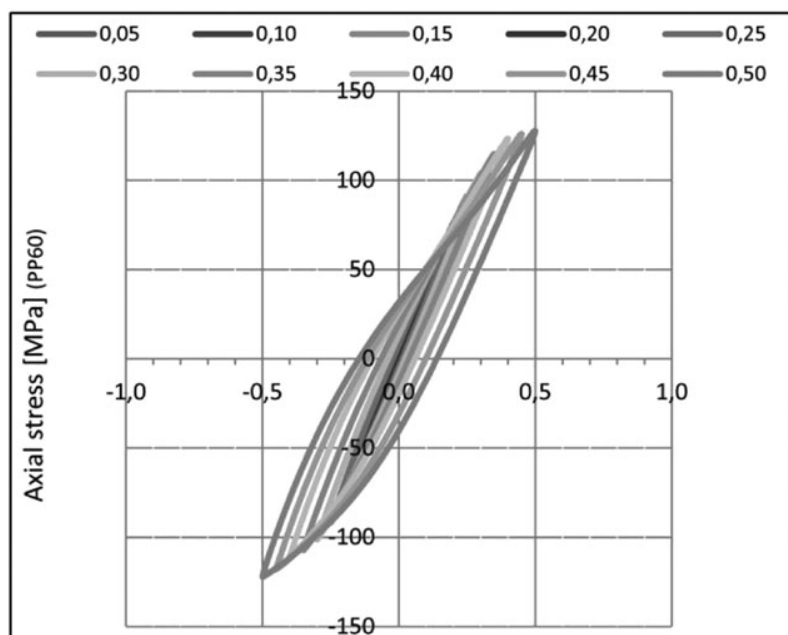

(A) Axial strain [\%] (PP60)

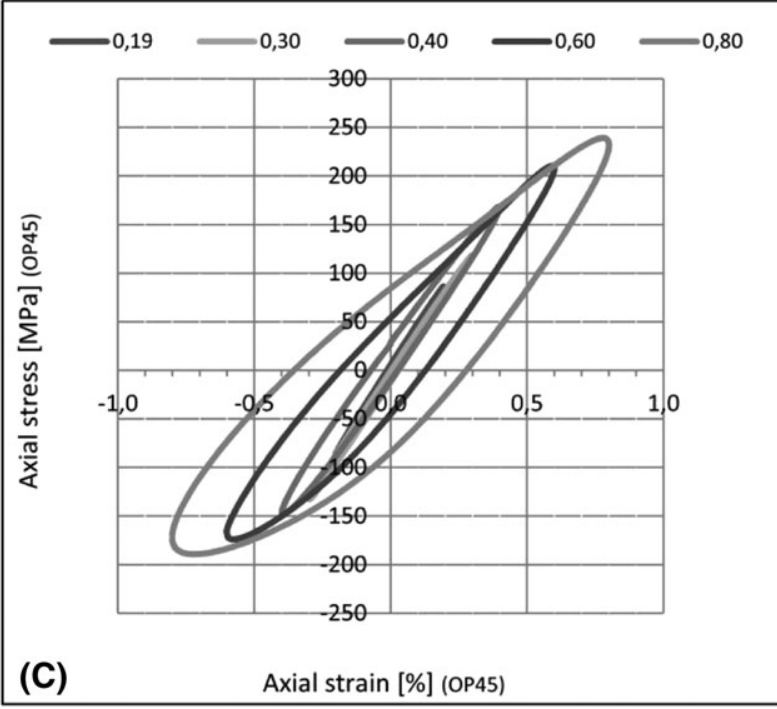

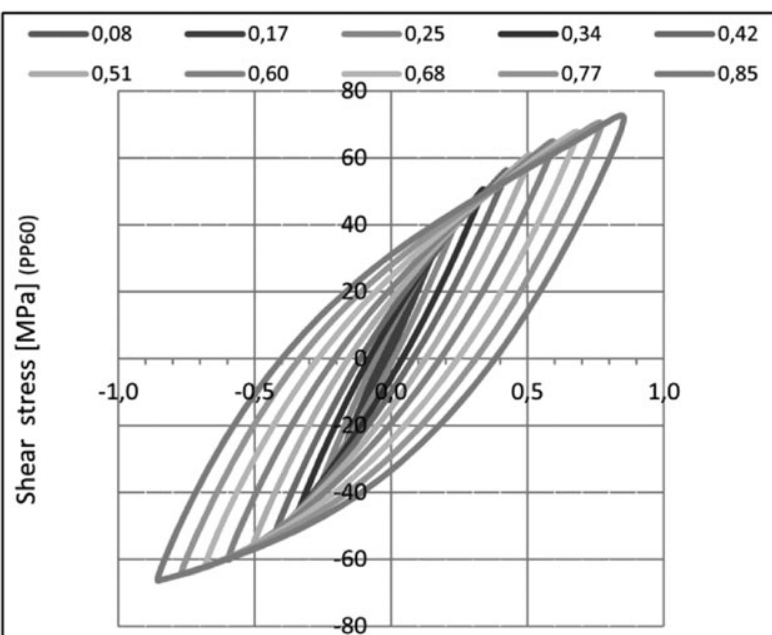

(B) Shear strain [\%] (PP60)

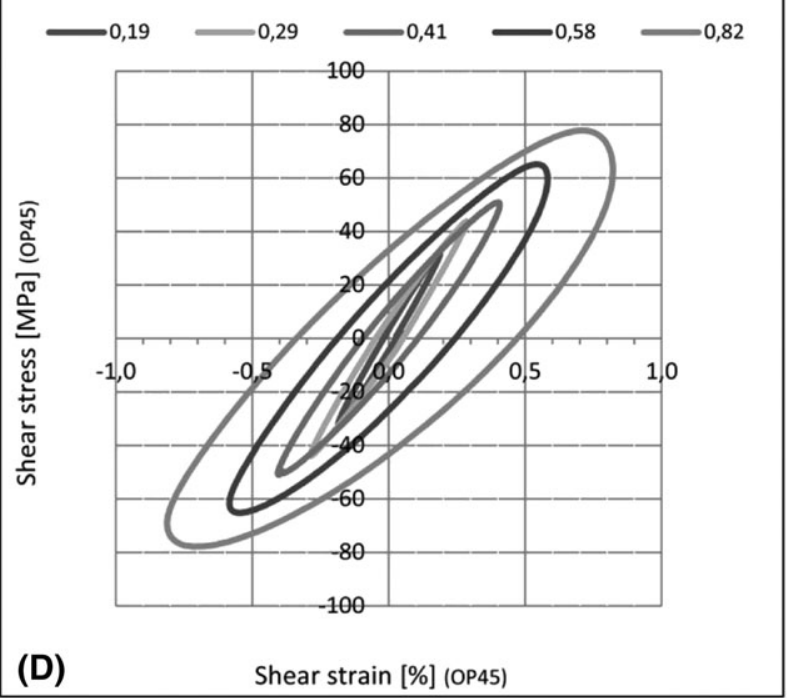

FIGURE 5 Experimental hysteresis loops for A, and B, Case 5, and C, and D, Case 6 
using the experimental data of points $4,3,2$, and 1 . The experimental data of these points ( 1 to 6 ) vary accordingly to the total strain and strain amplitude ratio given by $\lambda=\gamma / \varepsilon$. With these polynomials, it is possible to mimic the magnesium cyclic plastic factors such as twinning, de-twinning, and slip effects at each total strain level and strain amplitude ratios. The functions $P_{1}\left(\varepsilon_{s l}, \lambda\right)$ and $P_{4}\left(\varepsilon_{s l}, \lambda\right)$ depicted in Figure 1 estimate two yield stresses regarding the right and left side of the hysteresis loop for a given maximum total strain. The functions $P_{2}\left(\varepsilon_{s l}, \lambda\right)$ and $P_{5}\left(\varepsilon_{s l}, \lambda\right)$ estimate the plastic strains inherent to the maximum total strain, and the functions $P_{3}\left(\varepsilon_{s l}, \lambda\right)$ and $P_{6}\left(\varepsilon_{s l}, \lambda\right)$ estimate the back stresses.

Under biaxial loading conditions, it is obtained two hysteresis loops, one from the axial loading component and the other one from the shear loading. Therefore, it is obtained two different hysteresis loops which are dependent on each other. This dependence is captured by the strain amplitude ratio given by the shear strain to axial strain ratio $(\lambda=\gamma / \varepsilon)$, which is the angle in radians between the axial and shear strains amplitudes. The biaxial strain level is given by $\varepsilon_{s l}=\sqrt{\varepsilon_{t}^{2}+\gamma_{t}^{2}}$, which is a measure that can be directly related with the strain amplitude ratio. Thus, the axial and shear hysteresis loops of a biaxial loading for a given total strain can be given by Equation (1) for the axial loading component and Equation (2) for the shear one.

$$
\begin{aligned}
& \sigma_{\text {right }}\left(\varepsilon_{t}\right)=a_{\varepsilon_{t}} \varepsilon_{t}^{3}+\mathrm{b}_{\varepsilon_{t}} \varepsilon_{t}^{2}+\mathrm{c}_{\varepsilon_{t}} \varepsilon_{t}+\mathrm{d}_{\varepsilon_{t}} \\
& \sigma_{\text {left }}\left(\varepsilon_{t}\right)=e_{\varepsilon_{t}} \varepsilon_{t}^{3}+f_{\varepsilon_{t}} \varepsilon_{t}^{2}+g_{\varepsilon_{t}} \varepsilon_{t}+h_{\varepsilon_{t}} \\
& \tau_{\text {right }}\left(\gamma_{t}\right)=a_{\gamma_{t}} \gamma_{t}^{3}+\mathrm{b}_{\gamma_{t}} \gamma_{t}^{2}+\mathrm{c}_{\gamma_{t}} \gamma_{t}+\mathrm{d}_{\gamma_{t}} \\
& \tau_{\text {left }}\left(\gamma_{t}\right)=e_{\gamma_{t}} \gamma_{t}^{3}+f_{\gamma_{t}} \gamma_{t}^{2}+g_{\gamma_{t}} \gamma_{t}+h_{\gamma_{t}}
\end{aligned}
$$

The polynomial constants of Equations (1) and (2) are obtained with the Matlab polyfit function, which has as input the output values of the $\mathrm{P}$ functions described in Equations (3) to (6).

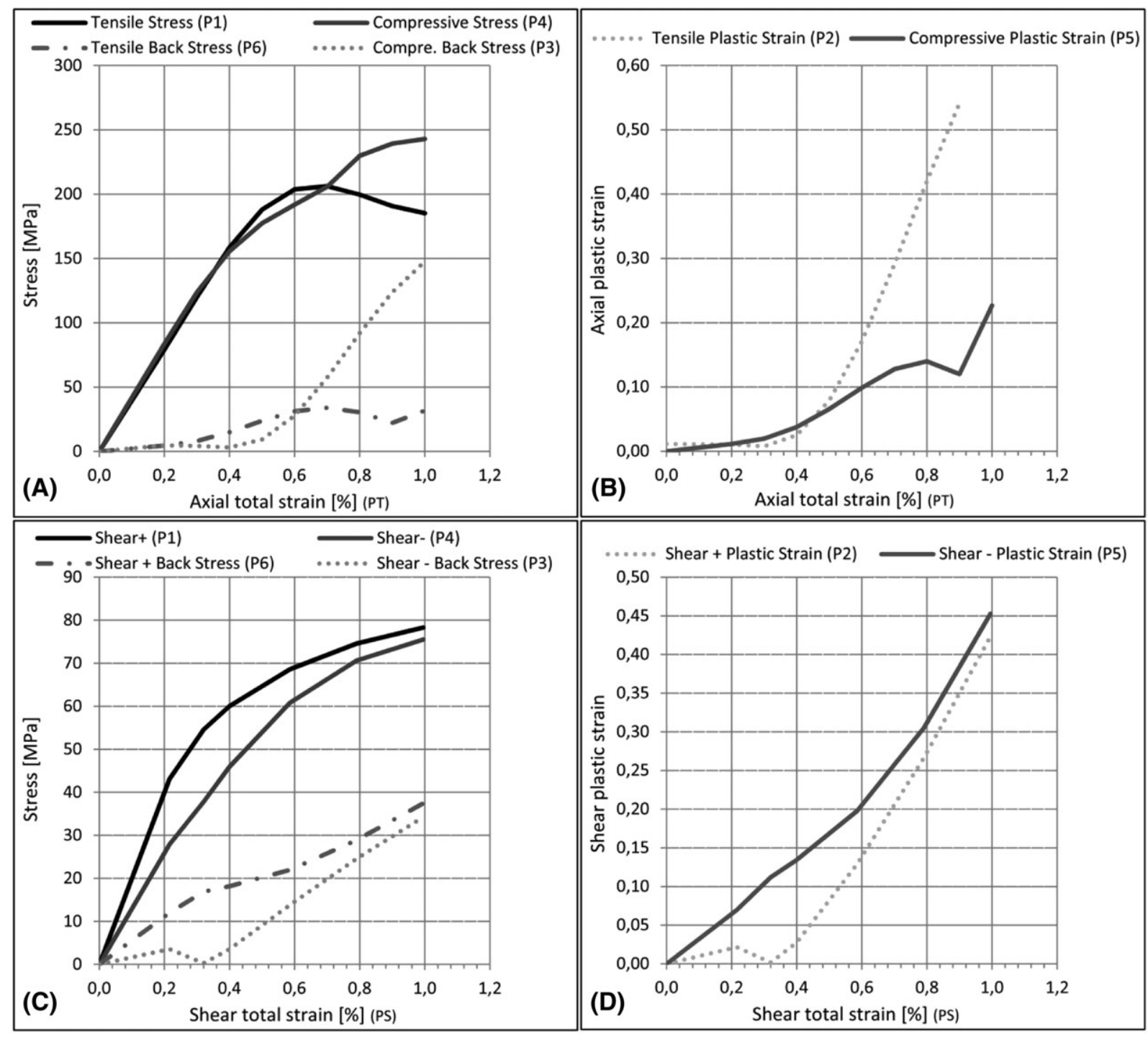

FIGURE 6 P point variation with total strain variation A, and B, Case 1; C, and D, Case 2 


$$
\begin{aligned}
{\left[a_{\varepsilon_{t}}, \mathrm{~b}_{\varepsilon_{t}}, \mathrm{c}_{\varepsilon_{t}}, \mathrm{~d}_{\varepsilon_{t}}\right]=} & \operatorname{polyfit}\left(P_{\text {axial }, 1}\left(\varepsilon_{s l}, \lambda\right), P_{\text {axial }, 2}\left(\varepsilon_{s l}, \lambda\right),\right. \\
& \left.P_{\text {axial }, 3}\left(\varepsilon_{s l}, \lambda\right), P_{\text {axial }, 4}\left(\varepsilon_{s l}, \lambda\right)\right)
\end{aligned}
$$

$$
\begin{aligned}
{\left[e_{\varepsilon_{t}}, \mathrm{f}_{\varepsilon_{t}}, \mathrm{~g}_{\varepsilon_{t}}, \mathrm{~h}_{\varepsilon_{t}}\right]=} & \operatorname{polyfit}\left(P_{\text {axial }, 4}\left(\varepsilon_{s l}, \lambda\right), P_{\text {axial }, 5}\left(\varepsilon_{s l}, \lambda\right)\right. \\
& \left.P_{\text {axial }, 6}\left(\varepsilon_{s l}, \lambda\right), P_{\text {axial }, 1}\left(\varepsilon_{s l}, \lambda\right)\right)
\end{aligned}
$$

$\left[a_{\gamma_{t}}, \mathrm{~b}_{\gamma_{t}}, \mathrm{c}_{\gamma_{t}}, \mathrm{~d}_{\gamma_{t}}\right]=\operatorname{polyfit}\left(P_{\text {shear }, 1}\left(\varepsilon_{s l}, \lambda\right), P_{\text {shear }, 2}\left(\varepsilon_{s l}, \lambda\right)\right.$,

$$
\left.P_{\text {shear }, 3}\left(\varepsilon_{s l}, \lambda\right), P_{\text {shear }, 4}\left(\varepsilon_{s l}, \lambda\right)\right)
$$

$$
\begin{aligned}
{\left[e_{\gamma_{t}}, \mathrm{f}_{\gamma_{t}}, \mathrm{~g}_{\gamma_{t}}, \mathrm{~h}_{\gamma_{t}}\right]=} & \operatorname{polyfit}\left(P_{\text {shear }, 4}\left(\varepsilon_{s l}, \lambda\right), P_{\text {shear }, 5}\left(\varepsilon_{s l}, \lambda\right),\right. \\
& \left.P_{\text {shear }, 6}\left(\varepsilon_{s l}, \lambda\right), P_{\text {shear }, 1}\left(\varepsilon_{s l}, \lambda\right)\right)
\end{aligned}
$$

Based on experiments, the P functions have the following shape under multiaxial loading conditions, see Equations (7) and (8):

$$
\begin{aligned}
P_{\text {axial }, i}\left(\varepsilon_{s l}, \lambda\right) & =a_{i}+b_{i} \varepsilon_{s l}+c_{i} \lambda+d_{i} \varepsilon_{s l}^{2}+e_{i} \lambda^{2}+f_{i} \varepsilon_{s l} \lambda \\
& +g_{i} \varepsilon_{s l}^{3}+h_{i} \lambda^{3}+i_{i} \varepsilon_{s l} \lambda^{2}+j_{i} \varepsilon_{s l}^{2} \lambda \\
P_{\text {shear }, j}\left(\varepsilon_{s l}, \lambda\right) & =a_{j}+b_{j} \varepsilon_{s l}+c_{j} \lambda+d_{j} \varepsilon_{s l}^{2}+e_{j} \lambda^{2}+f_{j} \varepsilon_{s l} \lambda \\
& +g_{j} \varepsilon_{s l}^{3}+h_{j} \lambda^{3}+i_{j} \varepsilon_{s l} \lambda^{2}+j_{j} \varepsilon_{s l}^{2} \lambda
\end{aligned}
$$

where constants with subscript $i$ and $j$ for axial and shear are obtained through numeric regressions of experimental stress-strain data. The subscript $i$ ranges from 1 to 6 and represents the six specific points identified in
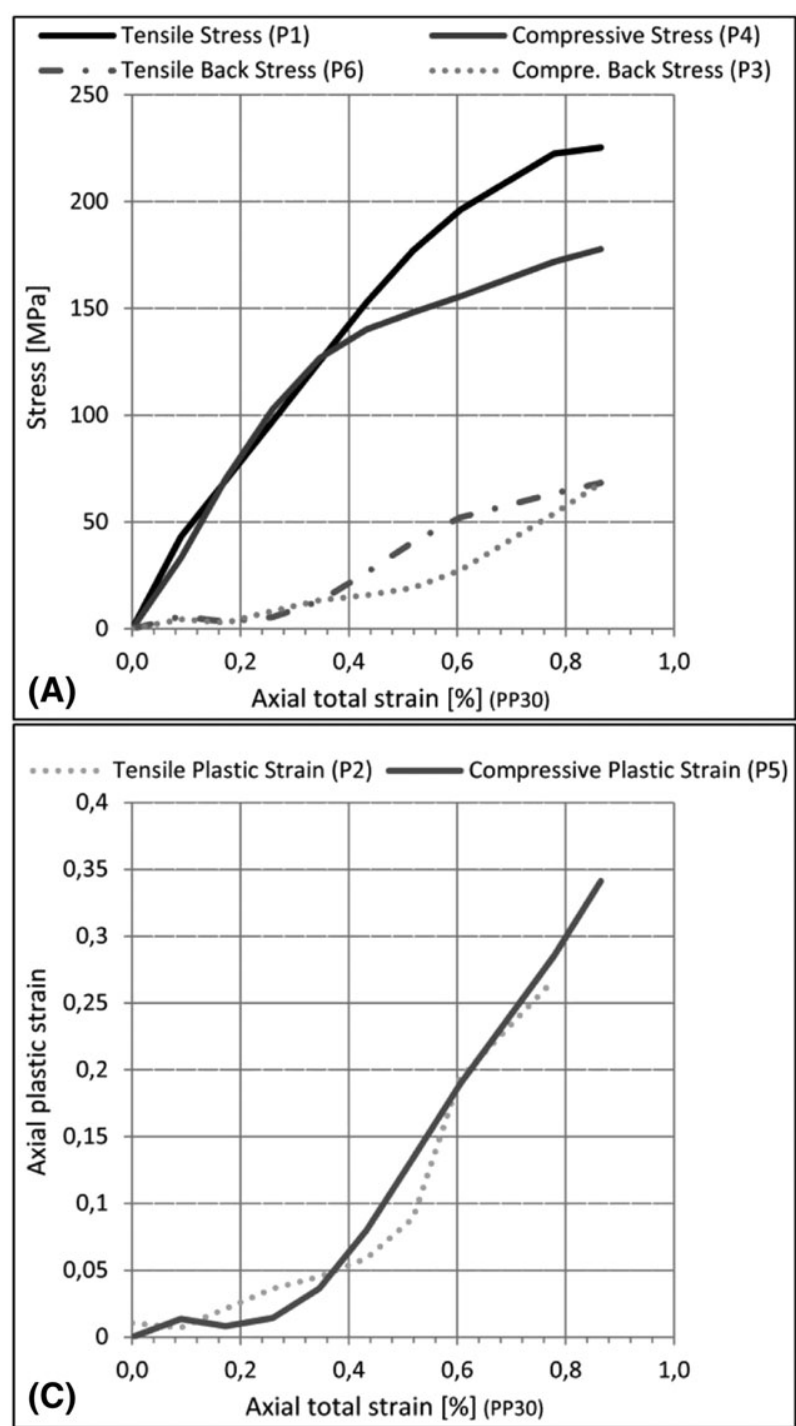
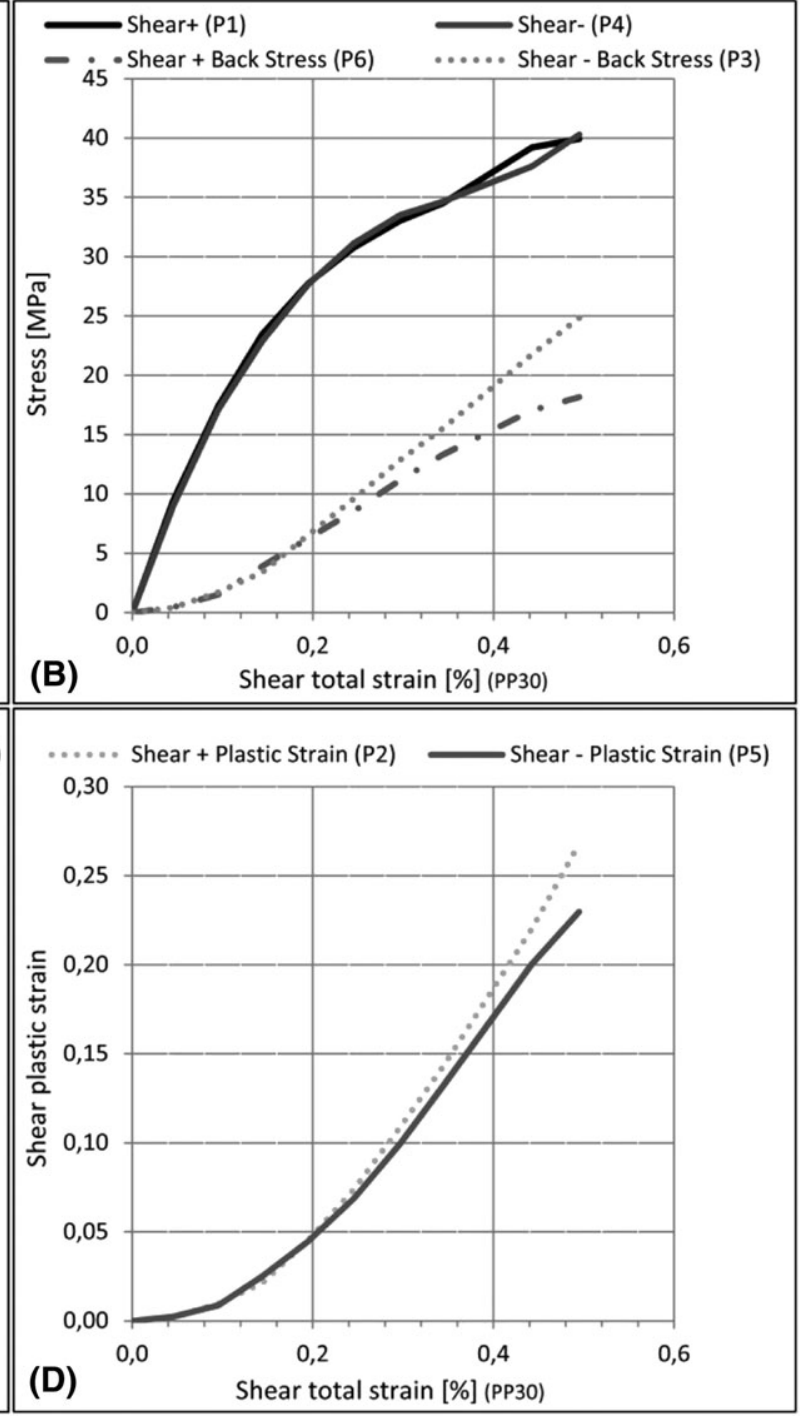

FIGURE 7 P point variation with strain variation for Case 3 
Figure 1 for the axial hysteresis loop, in the same way $j$ ranges from 1 to 6 and represents the six specific points for the shear hysteresis loop of a given loading.

\section{3 | MATERIALS AND METHODS}

\section{1 | AZ31B-F material}

The material used in this study was the AZ31B-F magnesium alloy. This material was acquired in the form of rods with $26 \mathrm{~mm}$ of diameter and $1000 \mathrm{~mm}$ in length. The rods were extruded in a temperature ranging from $360^{\circ} \mathrm{C}$ to $382^{\circ} \mathrm{C}$ with an extrusion speed of $50.8 \mathrm{~mm} / \mathrm{s}$. The applied extrusion ratio was about six, and after extrusion the alloy was air quenched. The tested specimens were machined in the extrusion/longitudinal direction and polished using decrease sandpaper grit to reach a mirror type finish. The specimen geometry and dimensions are presented in Figure 2. A biaxial servo-hydraulic testing machine was used to perform the cyclic tests under strain control with $\mathrm{R}=-1$; the loading shape in each loading channel (axial and shear) was in sinusoidal waveform. Several total strain amplitudes were considered and obtained at the same strain rate. The strain rate was about $0.003[1 / \mathrm{s}]$, which is a value lower than the limit, from which the strain rate affects the cyclic strain behaviour of magnesium alloys. The strain results were measured with a biaxial extensometer with a gauge length equal to $12.5 \mathrm{~mm}$.

The strain-controlled tests were performed considering total strains ranging from pure elastic to high cyclic plasticity, where collapse occurs at very few loading cycles. The total strains considered were the following: $0.1 \%$, $0.2 \%, 0.3 \%, 0.4 \%, 0.5 \%, 0.6 \%, 0.7 \%, 0.8 \%, 0.9 \%, 1 \%$, and $1.14 \%$. The experiments performed covers a realistic
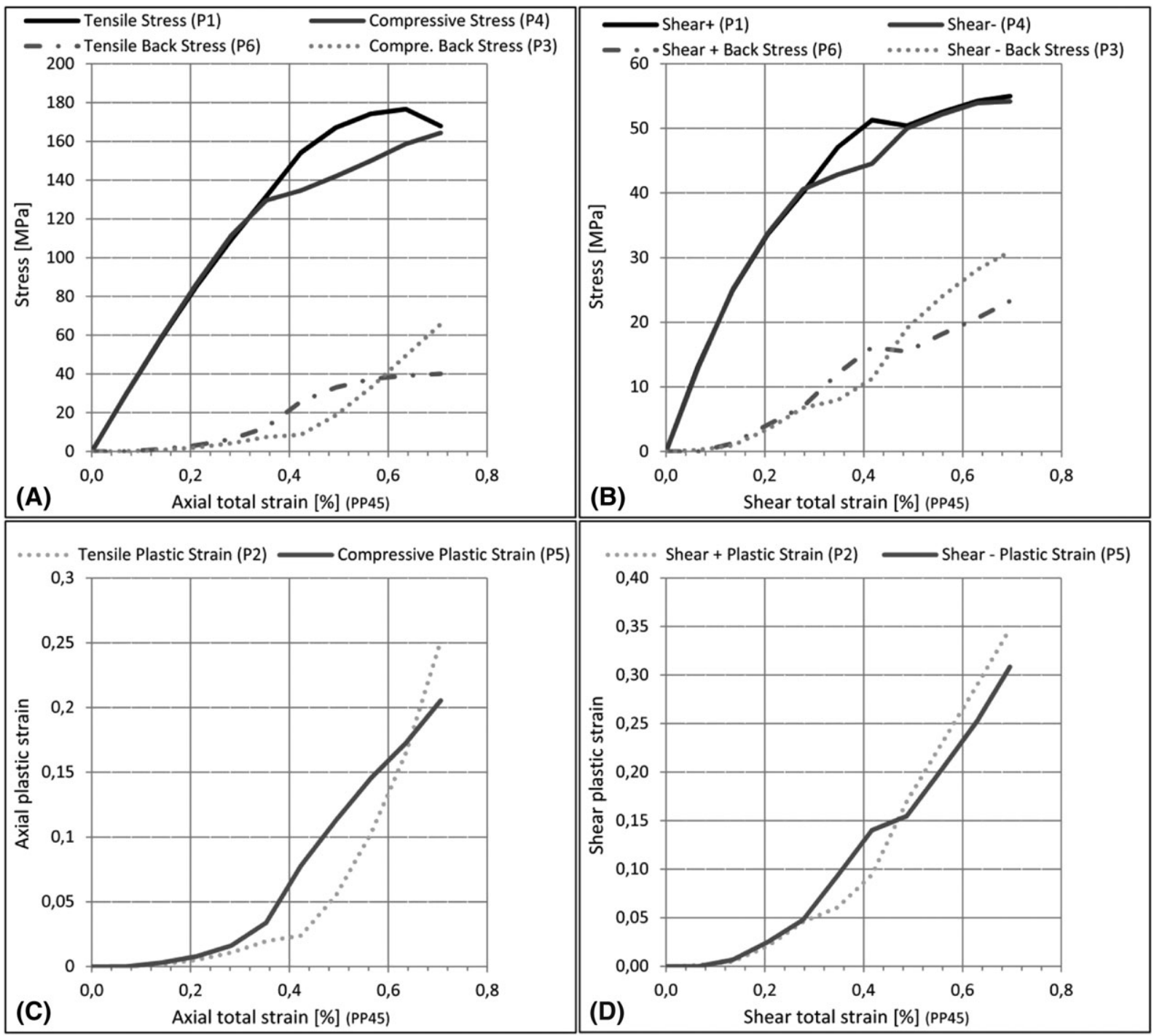

FIGURE 8 P point variation with strain variation for the Case 4 
stress-strain loading regime, ie, the need of cyclic mechanical behaviour for total strains higher that $1.14 \%$ is not very common in mechanical design; therefore, the total strain range considered is adequate for design activities. Moreover, the total strain values and loading paths were selected in order to perform valid numeric regressions. Because the phenomenological model is based on experiments; thus, it will be only valid within the total strain range considered, in this case from $0.1 \%$ to $1.14 \%$. A total of 12 specimens were tested, two specimens for each loading case described in Figure 3. The tests were performed in an incremental-step approach where loading blocks of 10 cycles (one block per strain level) were applied until reach rupture. During experiments, each cyclic test was considered concluded at the specimen total separation. The average total number of loading cycles at rupture for each loading case described in Figure 3 was
$N f_{\text {Case } 1}=63, N f_{\text {Case } 2}=76, N f_{\text {Case3 }}=94, N f_{\text {Case4 }}=109$, $N f_{\text {Case } 5}=103$, and $N f_{\text {Case6 }}=57$.

\section{2 | Biaxial loading paths}

To evaluate the AZB-F mechanical behaviour, six biaxial loading paths, described in Figure 3, were considered. These loading paths were previously studied by the present authors to capture anisotropic fatigue proprieties. ${ }^{15}$ The first loading case, Case 1, is a pure uniaxial tensile test, case PT. The second one, Case 2, is a pure shear loading, named as case PS. Cases 3, 4, and 5 are proportional loadings with SAR equal to $30^{\circ}, 45^{\circ}$, and $60^{\circ}$, respectively. Finally, Case 6 is a nonproportional loading case, with a SAR equal to $45^{\circ}$ and a phase shift equal to $90^{\circ}$. All these loading paths were implemented in experiments and
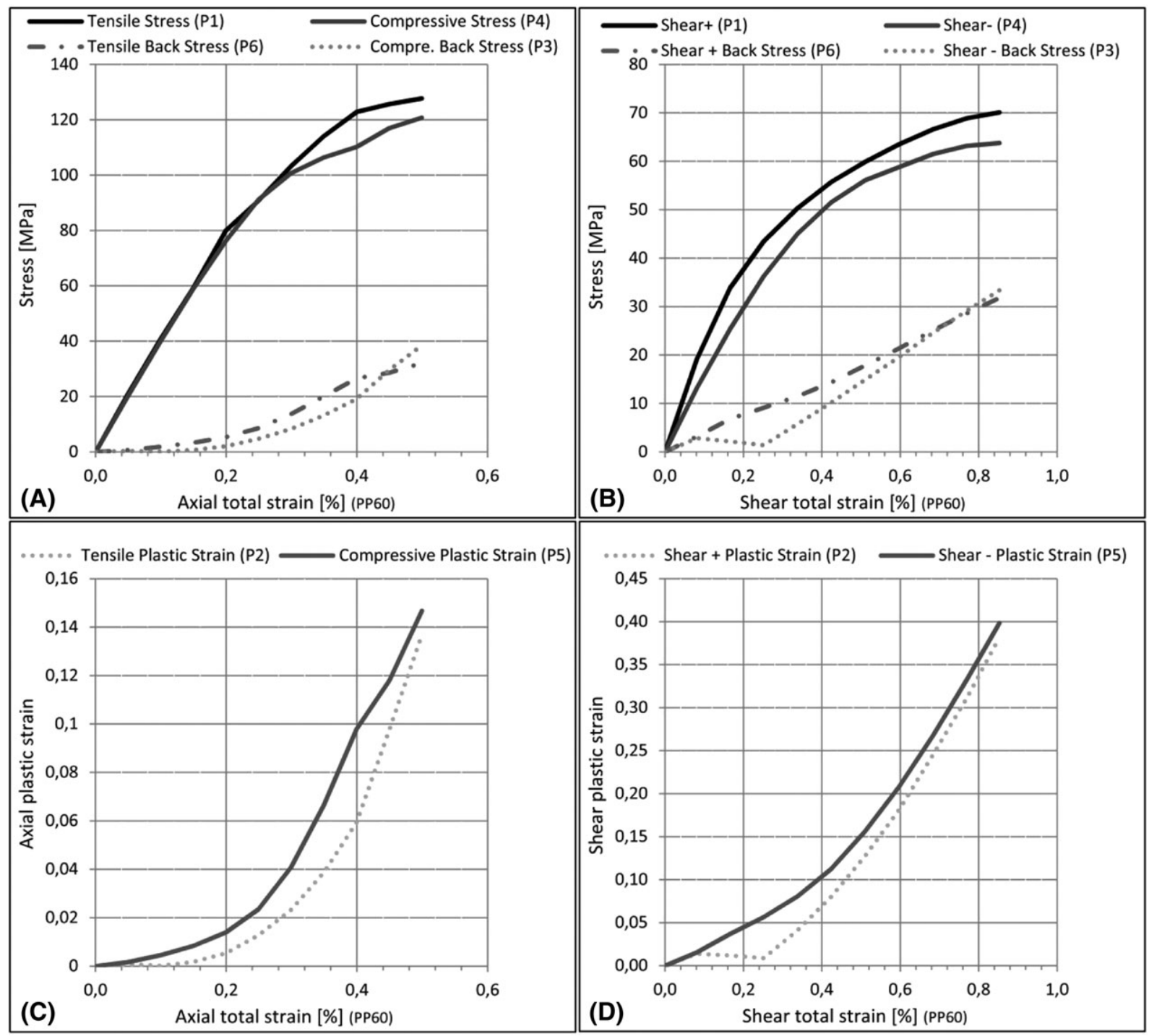

FIGURE 9 P point variation with strain variation for Case 5 
considered in the developed phenomenological approach. The specimens were tested at room temperature and ended when the specimens were totally separated.

\section{4 | RESULTS AND DISCUSSION}

Figure 4A,B presents the uniaxial results for the hysteresis loops in pure axial and pure torsion loading conditions, ie, Case 1 (PT) and Case 2 (PS). For the PT loading case, Figure 4A, it can be concluded that the hysteresis loops are not symmetric, having different plastic strains in tension and compression. Moreover, the axial tension, for the same amplitude of total strain, is different from the compression one. Also, the plastic strains and stresses in tension and compression vary nonlinearly. These results show the presence of six independent parameters in a magnesium alloy's hysteresis loop that are independent from each other, namely the $\mathrm{P}$ points presented in Equations (7) to (8).

Figure 4B shows the hysteresis loops for the AZ31B-F magnesium alloy in pure torsion being quite symmetric, which is a mechanical behaviour very different from the axial one, ie, the shear stresses have the same absolute value for the same shear strain limits (considering a strain-control approach). Moreover, the shear plastic strains are also very similar, the difference between plastic strain (left-right) that can be seen in Figure 4B derived from the direction of the first loading cycle.

Figure 4C,D shows the axial and shear hysteresis loops resulted from a proportional biaxial loading with a SAR equal to $30^{\circ}$ (Case 3 ). In this case, the axial strain component is bigger than the shear one, resulting in shear stress amplitude lower than the axial one. Therefore, the axial
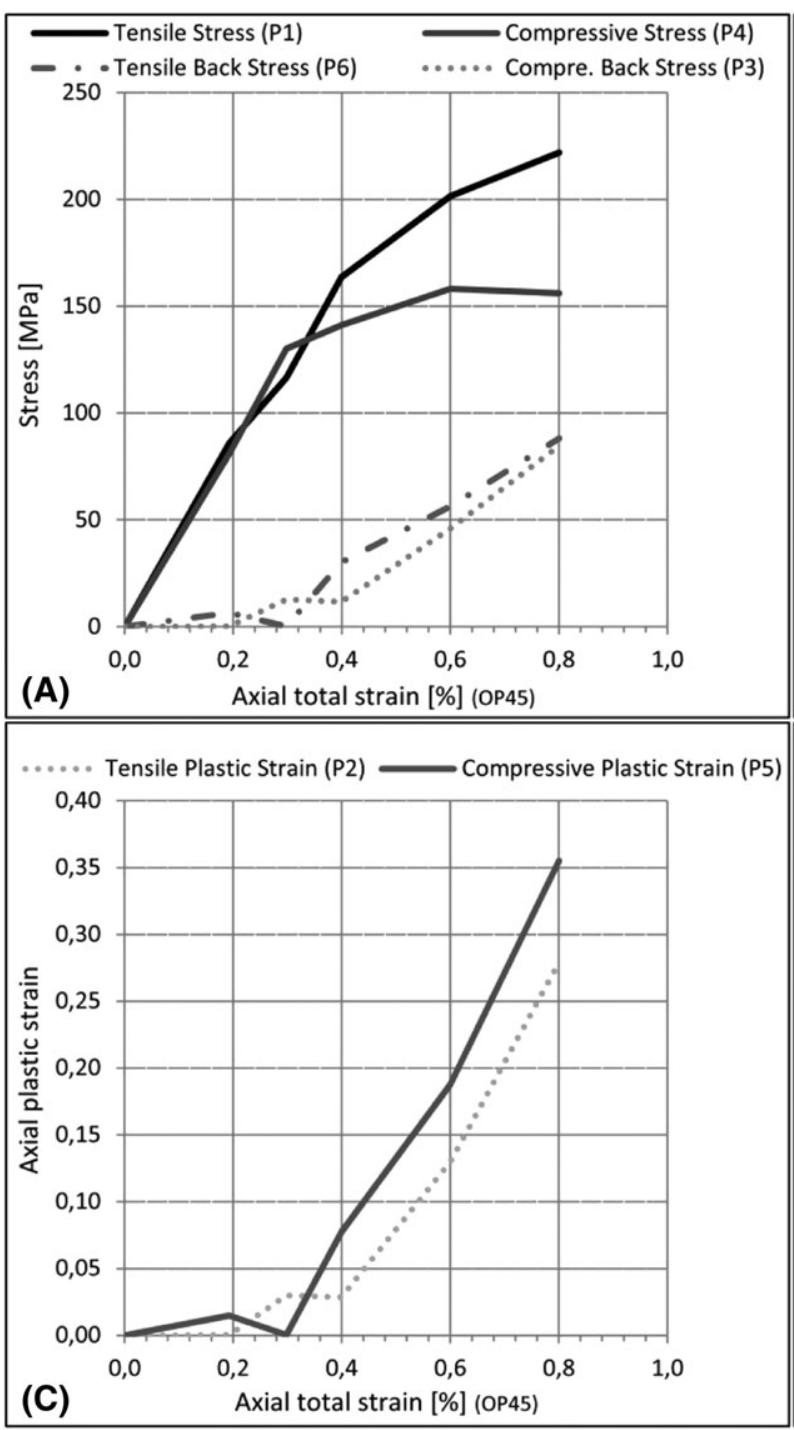
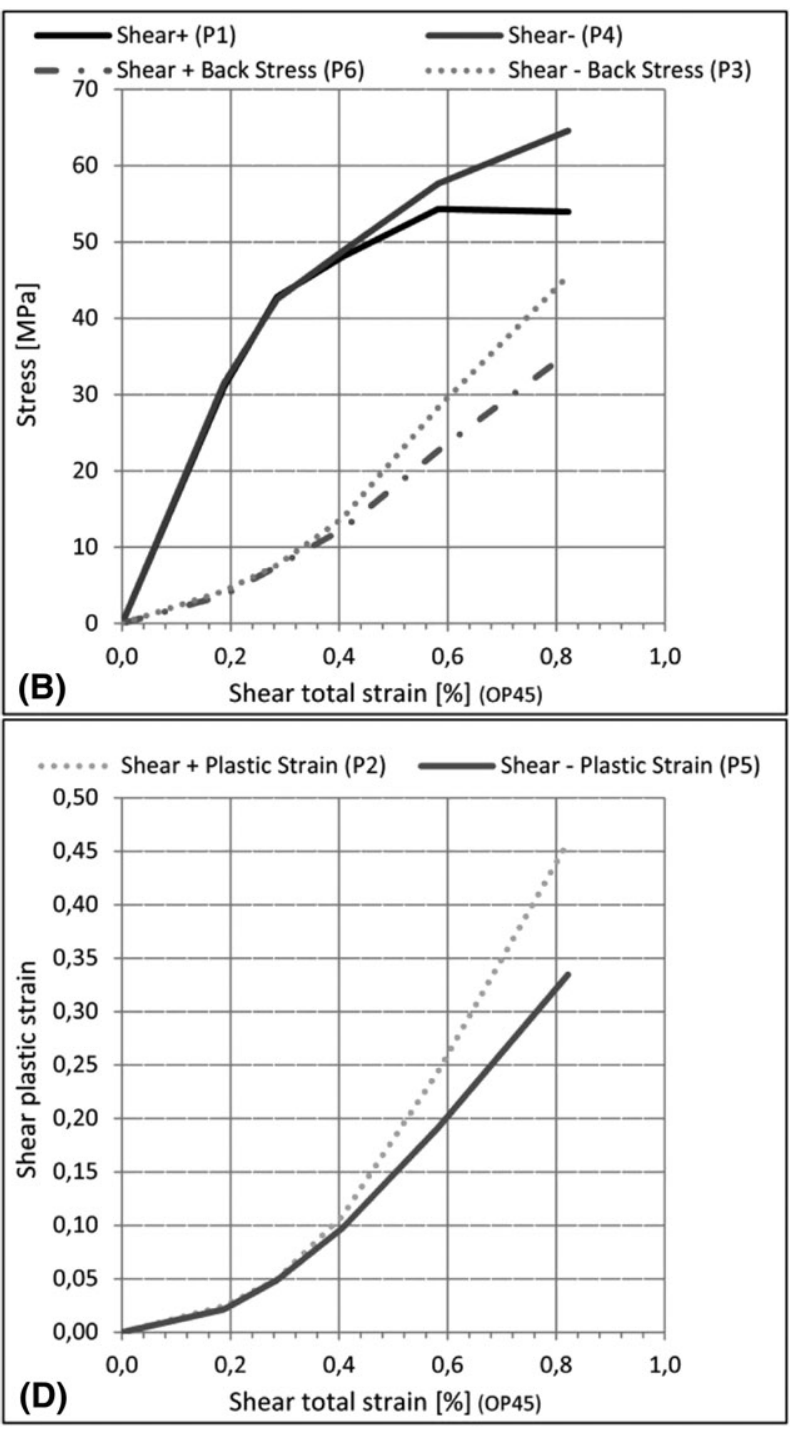

FIGURE 10 P point variation with strain variation for Case 6 
deformation and inherent plastic deformation govern the deformation behaviour. Figure 4E,F shows the stress strain results for Case 4 , a biaxial loading with SAR equal to $45^{\circ}$, where the maximum amplitude of the axial and shear strains are equal. Despite the equality between shear and axial strain amplitudes, the resulted stresses have amplitudes very different. For instance, at 0.5\% shear strain, the respective shear stress amplitude is $50 \mathrm{MPa}$, but the axial strain at $0.5 \%$ leads to $160 \mathrm{MPa}$. Moreover, the axial stress is quite different in compression and tension, even for the same strain amplitude in tension and compression. The shear strain hysteresis loops for Case 4, Figure 4F, also show a symmetric pattern, which means that the shear mechanical response of AZ31B-F is not affected by the stress amplitude ratio (SAR), because it has the same pattern found in the pure shear loading case (uniaxial). Comparing both Cases 3 and 4 , one can conclude that increasing the SAR value reduces the axial plastic strain and increases the shear one; therefore, the SAR increase implies a predominance of shear effects.

Figure 5A,B presents the experimental results for the proportional loading with SAR equal to $60^{\circ}$. It is quite evident that the shear hysteresis loops have bigger plastic strains than the axial ones. These results confirm that increasing the SAR values decreases the axial strain component and increases the shear one; therefore, the deformation mechanisms and the inherent stresses are very different for each SAR. Thus, the typical equivalent stress concept, such as the von Mises equivalent stress/strain, is unsuitable to modulate this type of deformation effects, because equivalent stress/strain approaches are independent of the loading direction. For example, it can be obtained the same von Mises equivalent stress for all
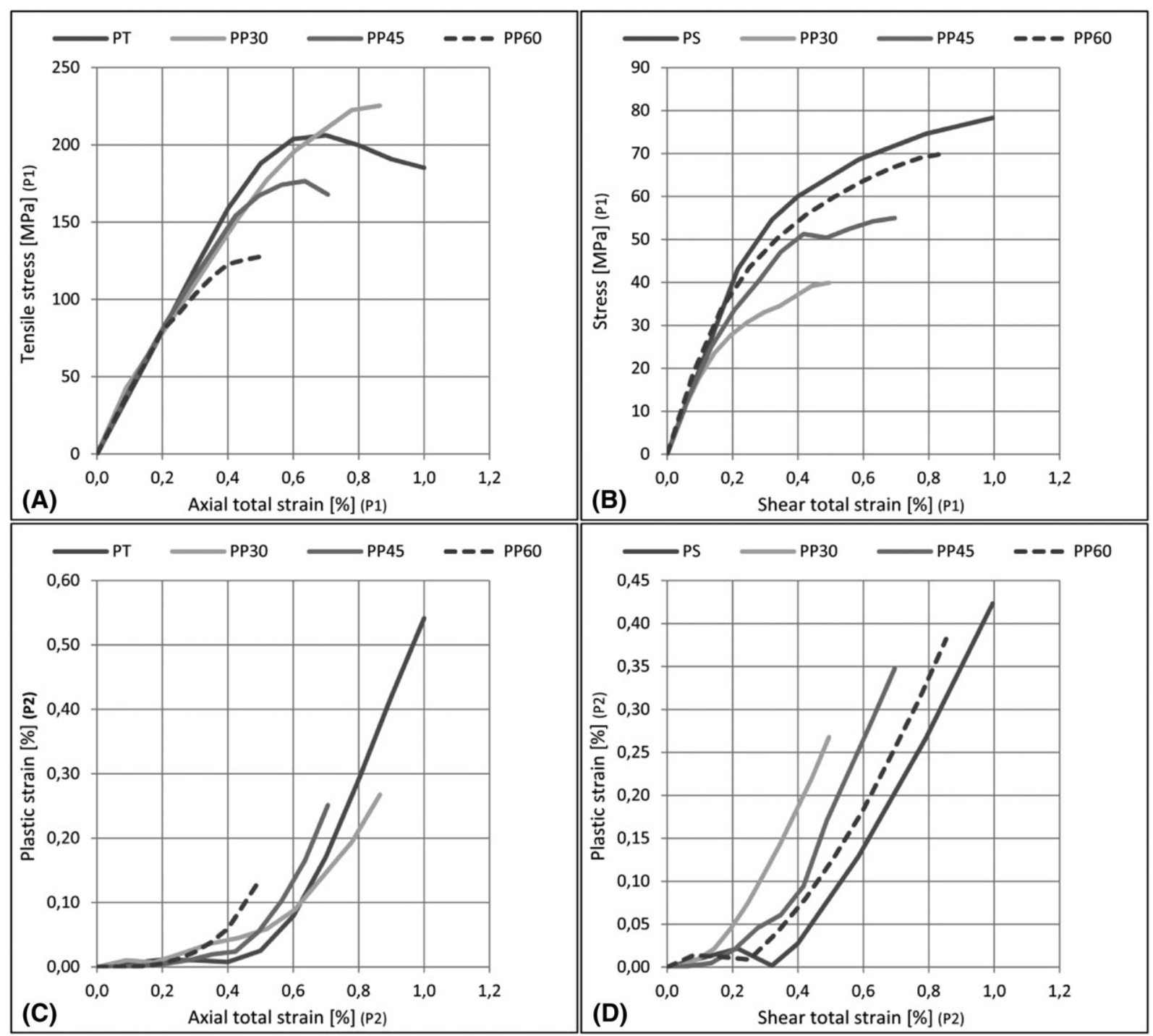

FIGURE 11 P1 and P2 variation with SAR and strain variation-Proportional loadings 
possible SARs considered here, ie, for all loading directions, but each SAR has different deformation behaviour as is seen in this work.

Clearly, nonproportional loadings create a different loading pattern in the material elastic-plastic cyclic behaviour. Comparing proportional and nonproportional loadings with the same SAR $\left(\lambda=45^{\circ}\right)$ and same maximum loading amplitudes, see Figures 4C,D and 5C,D, it can be concluded that the plastic strains and back stresses have quite different values in each loading path. These two parameters, plastic strain and back stresses, are two indicators of the loading type effect on the material response to the loading path. Correlating the stabilized hysteresis loops shown in Figures 4 and 5 with the AZ31B-F monotonic curve in axial and shear, it was possible to conclude in all loading cases, that for the axial loading component, there is a hardening in tension and a slight softening in compression, and for the shear loading component it was shown a slight hardening in both loading directions.

Figure 6 shows the variation of the P points for Case 1 and Case 2. The curves represent the $\mathrm{P}$ point variation in function of strain level. Figure 6A presents the stresses in each hysteresis loop for each stress amplitude level. In Figure 6A,B, the graph's abscissa represents the axial strain amplitude of the cyclic load, which in experiments takes negative and positive values. Therefore, tensile stresses result from positive strains and compressive strains result from negatives stresses. To better interpret and correlate the results of compression and tension loads, it is considered here that the compressive strains and inherent stresses are positive. In this way, it is possible to verify for loading case PT (Case 1) that the stressstrain relation in tension and compression is almost equal
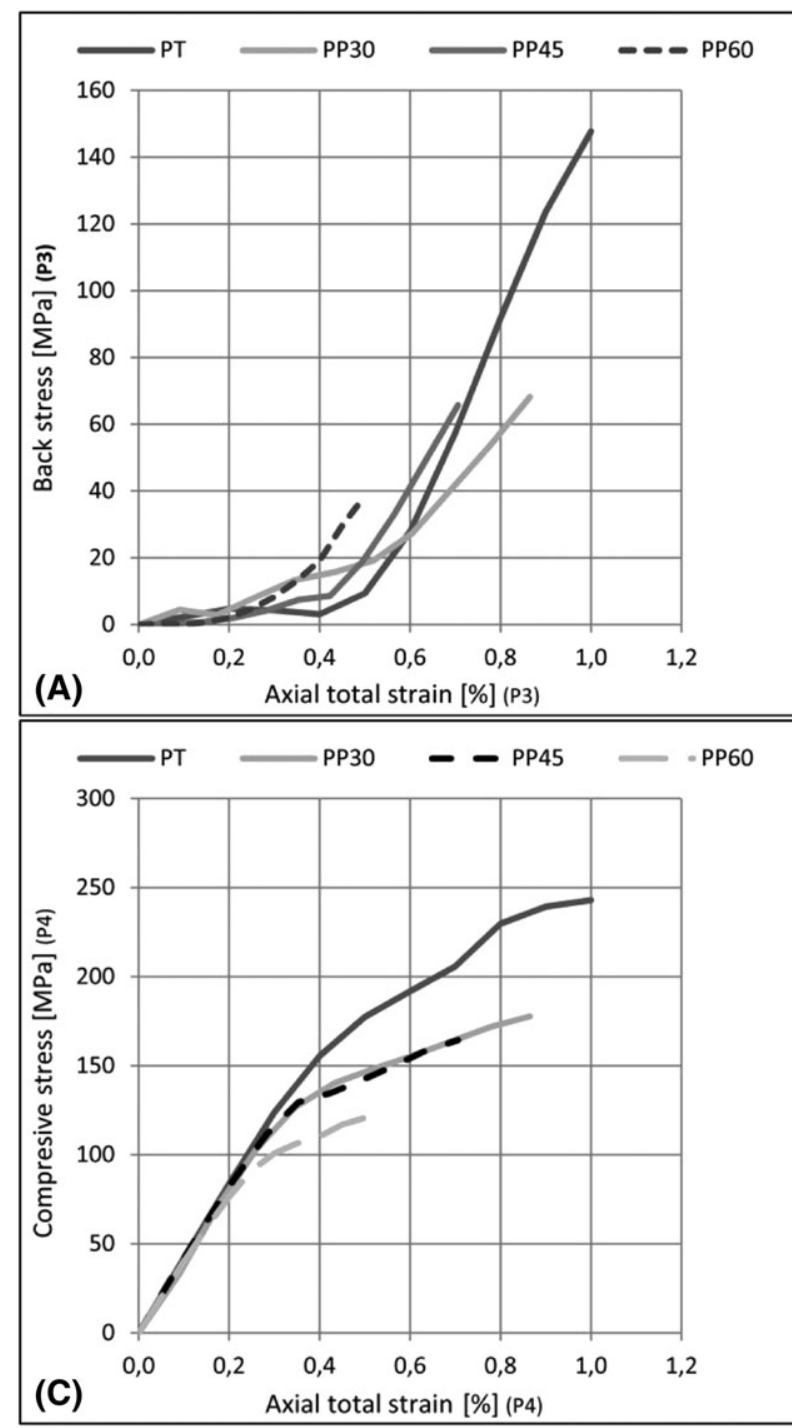
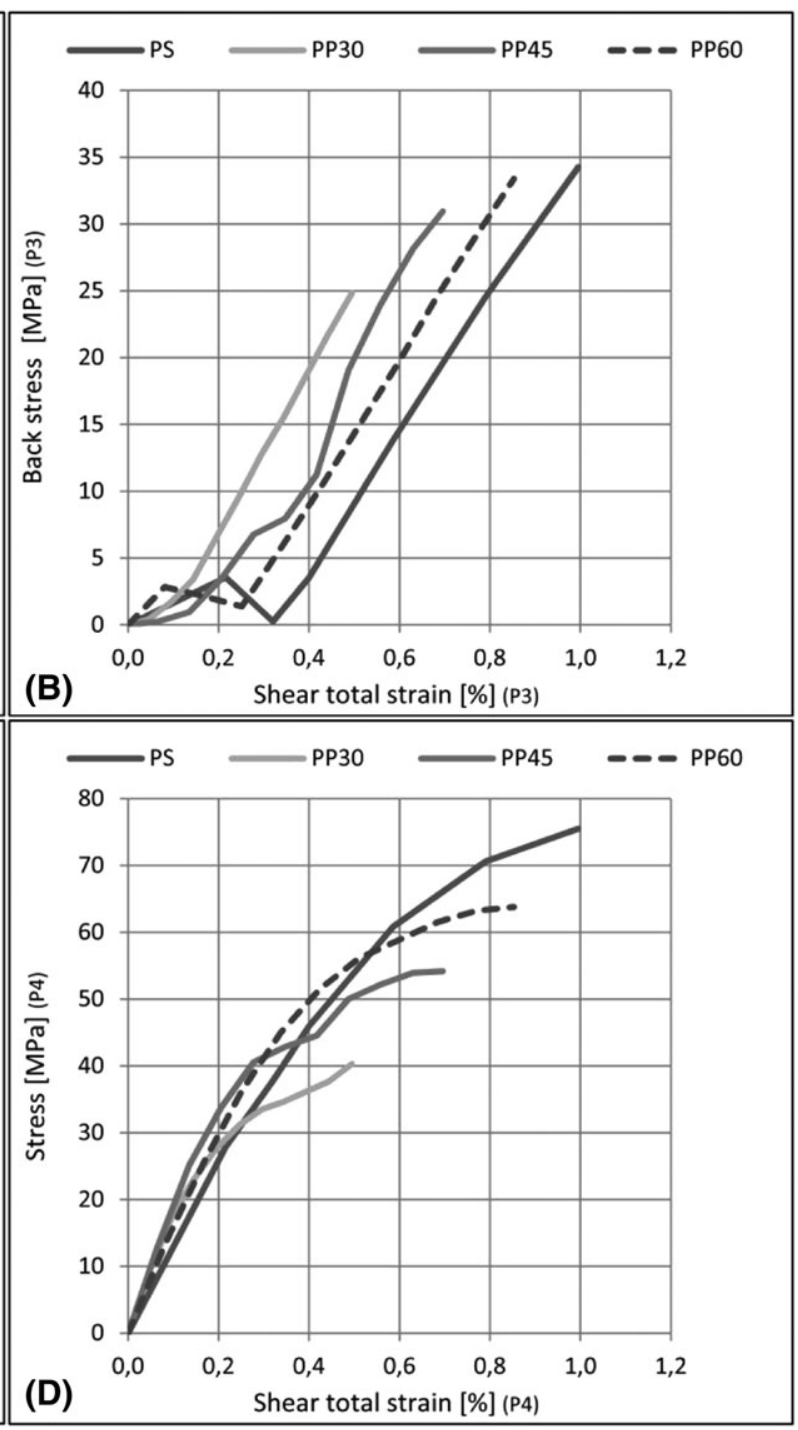

FIGURE 12 P3 and P4 variation with SAR and strain variation 
for total strains below $0.4 \%$. But, beyond that value, the cyclic elastic-plastic behaviour is quite different in tension and compression, see Figure 6A,B.

Each line presented in these graphs (see Figures 6 to 12) represents the variation of the $P$ points with the strain amplitude level variation (total strain). Making a fitting of these experimental results, it allows to get functions that capture the $\mathrm{P}$ point variation according to the total strain amplitude; therefore, in this way, it becomes possible to estimate any hysteresis loop.

Figure 6C,D presents the results for the Case 2, the pure shear loading case, ie, PS. Observing these results, and contrary to Case 1, PT, the stresses inherent to the shear stress amplitudes are always different in all shear strain levels. The same can be observed for the back-stress evolution as well as plastic strain. This can be explained by the first loading direction of the shear strain, where the first direction of the plastic shear strain influences the overall elastic-plastic deformation.

Figure 7 presents the results for the loading case PP30, Case 3, previously identified in Figure 3. Here, it is presented a proportional loading case with SAR equal to $30^{\circ}$. Figure 7A,C shows the results for the axial component of the proportional loading, and Figure 7B,D shows the shear one.

Similarly to the pattern observed in PT loading case, the PP30 axial elastic-plastic stresses are quite similar until reach $0.4 \%$ of total axial strain. Surprisingly, the shear stress evolution has the same stress values in both loading directions; the same can be said for plastic strains.

It can be concluded that the hysteresis loops of the shear component of the proportional loading path (PP30) are symmetric for all strain levels considered.
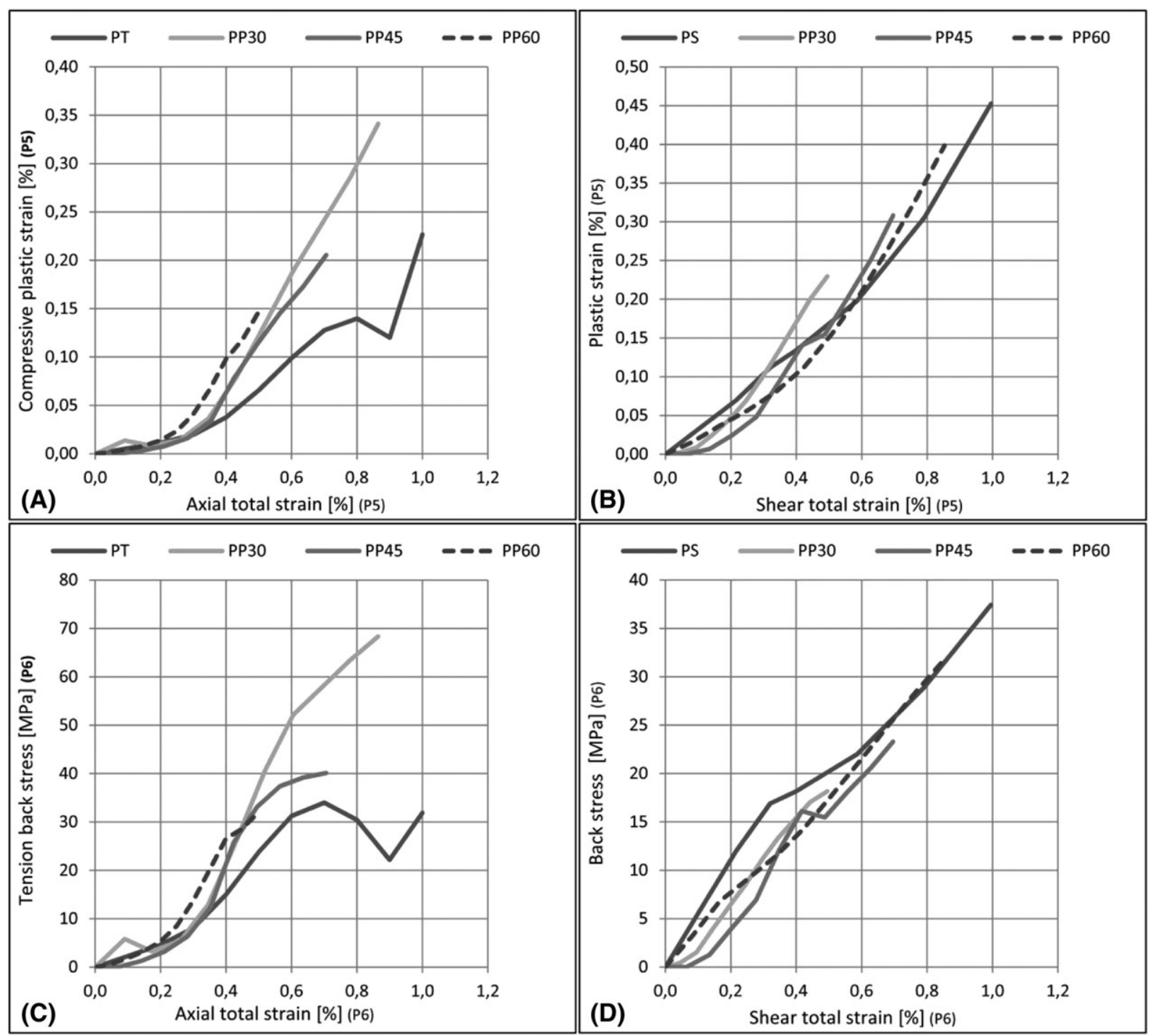

FIGURE 13 P5 and P6 variation with SAR and strain variation 
Figure 8 shows the cyclic behaviour of the magnesium alloy, AZ3B-F, under a proportional loading path with SAR equal to $45^{\circ}$. The same pattern found in PP30 could be seen here in PP45 loading case; the compression and tension begin to have different stresses beyond the $0.4 \%$ shear strains. The shear hysteresis loops also have a symmetric trend, as seen in the previous loading paths. In this loading path, PP45, the axial and shear strains have the same amplitude, but the stresses inherent to these amplitudes are quite different. For instance, for $0.4 \%$ of total strain, the yield stress in axial is about $140 \mathrm{MPa}$, and the yield stress in shear is about $50 \mathrm{MPa}$.

Figure 9 shows the results for the PP60 loading case, which is a proportional loading with a SAR equal to $60^{\circ}$. In this case, the shear component is greater than the axial one. The axial cyclic pattern is maintained in this loading path (PP60), but the shear loading starts to show shear strain dominance in the cyclic plastic behaviour.

For instance, Figure 9B has the same pattern seen in the pure shear strain loading case, Case 1, PS, ie, the two strain-stress curves (two torsion directions) are not coincident; in this case, the first loading direction dominates the cyclic elastic-plastic behaviour.

Figure 10 shows the results for the nonproportional loading case; these results are lightly different from the
PP45 loading case. The nonproportional cyclic deformation has a huge influence in the stress-strain pattern, but in terms of back stresses and plastic strain values, the differences from PP45 loading case are not so evident.

Figure 11 shows the variation of points $\mathrm{P} 1$ and $\mathrm{P} 2$ for all proportional loading cases considered here. It is presented the P1 and P2 variation for the axial and shear total strain at each proportional loading case. Because the variations of P1 and P2 are not coincident for all loading paths, it can be concluded that the SAR variation has an influence in the hysteresis loops and in the inherent cyclic elastic-plastic behaviour.

Moreover, in the axial direction, the $\mathrm{P} 1$ variation is quite similar for all loading paths at total strains below $0.4 \%$. However, for the P2 values, only below $0.2 \%$, it can be considered such simplification.

Figure 12 shows the variation of $\mathrm{P}$ points $\mathrm{P} 3$ and $\mathrm{P} 4$. The $\mathrm{P} 3$ evolution in the shear component is quite different in all loading paths, see Figure 12B. The P3 axial evolution until reaches $0.4 \%$ has a similar pattern, but for the shear one it seems that there is no pattern. For P4, Figure $12 \mathrm{C}, \mathrm{D}$, it can be considered that below $0.4 \%$ of total strain, both axial and shear have similar evolution pattern in both loading paths.

Figure 13 presents the experimental results for $\mathrm{P}$ points P5 and P6. It seems, as seen in the other P point
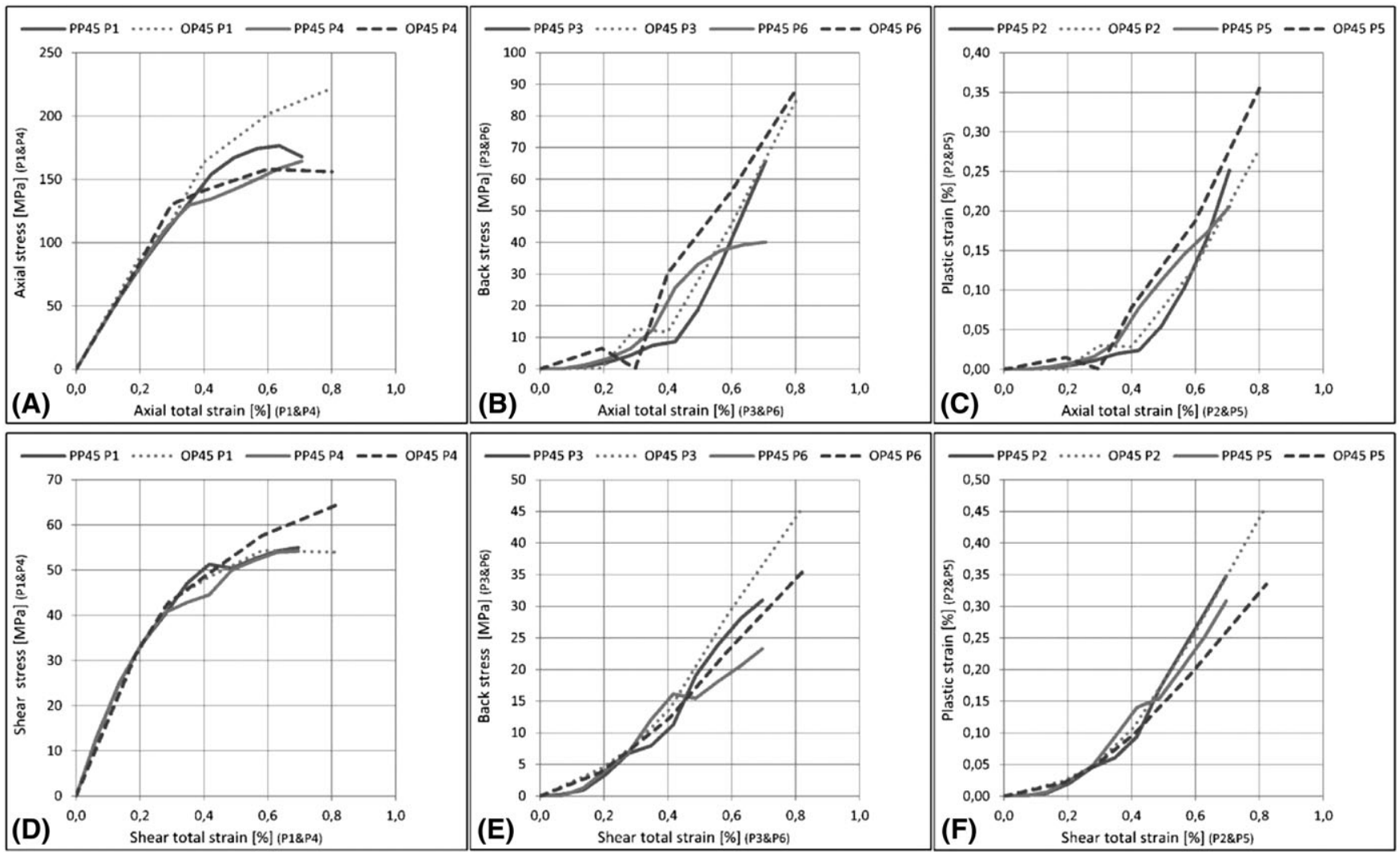

FIGURE 14 P point variation in Case 4 and Case 6 
analysis, that they can be fairly merged as one, but only below of $0.4 \%$ of total strain, but beyond that value, they present values completely different, for each loading case.

From these results, it can be concluded that magnesium hysteresis loops can be fairly estimated using the uniaxial results if the total strain in shear and axial are below the upper limit of $0.4 \%$. For greater total strains, this conclusion is not valid, and a multiaxial mapping for the cyclic behaviour is needed.

Figure 14 presents a comparison between $\mathrm{P}$ points of proportional and nonproportional loading paths, PP45 and OP45, respectively; both loading paths have a SAR equal to $45^{\circ}$. The $\mathrm{P}$ points, of the shear loading components, have an evolution very similar; however, it is in

TABLE 1 AZ31B-F polynomial constants for the P functions for proportional loadings (axial component) valid for $\lambda\left[0^{\circ} ; 90^{\circ}\right]$ and $\varepsilon_{s l}[0 ; 1]$

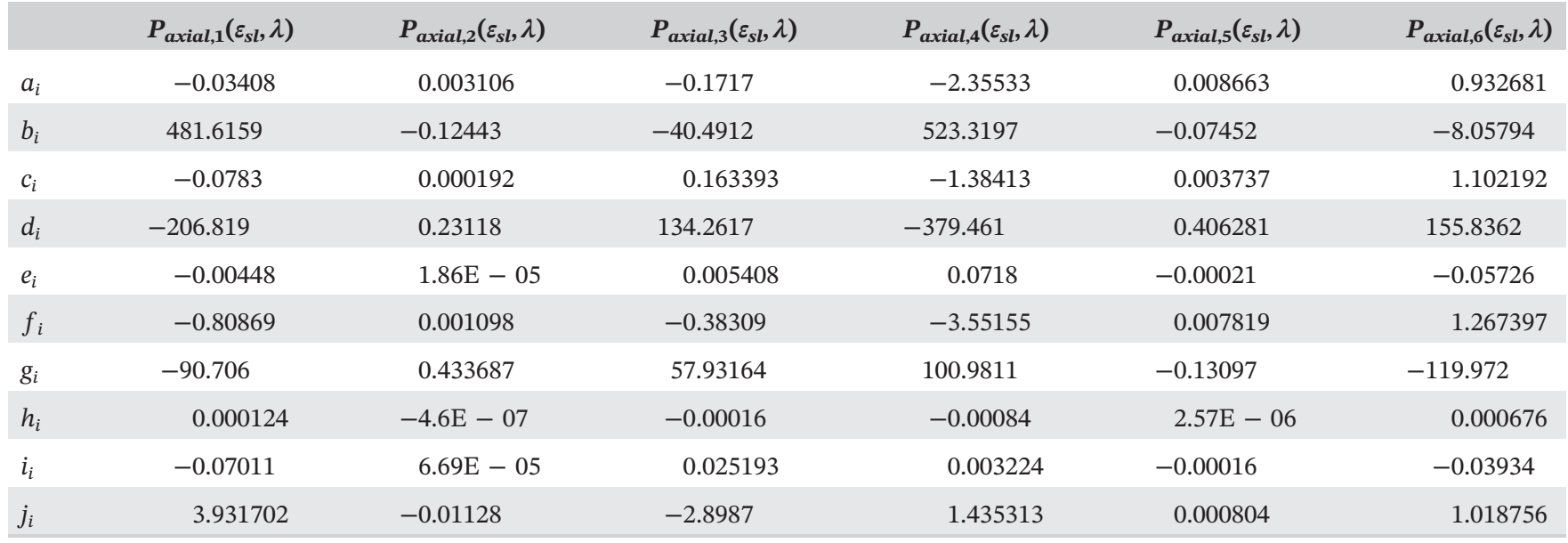

TABLE 2 AZ31B-F polynomial constants for the P functions for proportional loadings (shear component) valid for $\lambda\left[0^{\circ} ; 90^{\circ}\right]$ and $\varepsilon_{s l}[0 \sim 1]$

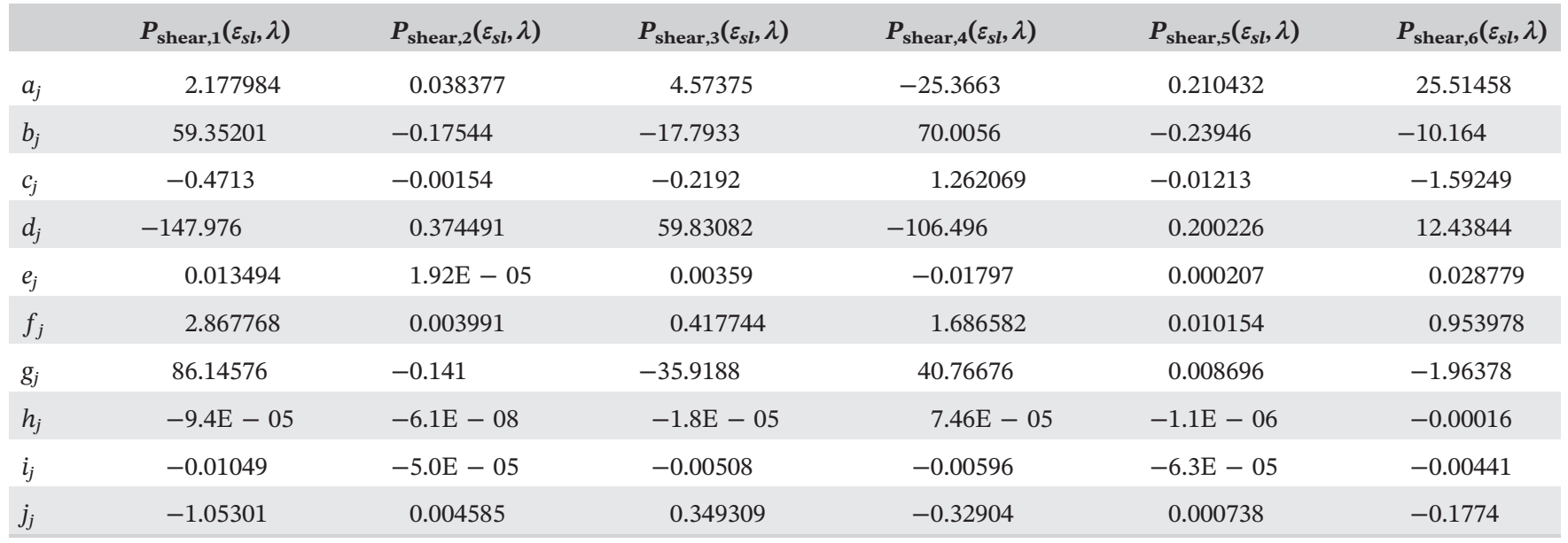

TABLE 3 Polynomial constants for the P functions for proportional loadings

\begin{tabular}{|c|c|c|c|c|c|c|c|c|}
\hline & $P_{1}[M P a]$ & $\boldsymbol{P}_{2}[\varepsilon]$ & $P_{3}[M P a]$ & $P_{4}[\mathrm{MPa}]$ & $\boldsymbol{P}_{5}[\varepsilon]$ & $P_{6}[M P a]$ & & \\
\hline \multirow[t]{2}{*}{ Shear } & 33.19 & 0.10 & 12.41 & 34.40 & 0.09 & 10.28 & & \\
\hline & $a_{\varepsilon_{t}}$ & $b_{\varepsilon_{t}}$ & $c_{\varepsilon_{t}}$ & $d_{\varepsilon_{t}}$ & $e_{\varepsilon_{t}}$ & $f_{\varepsilon_{t}}$ & $g_{\varepsilon_{t}}$ & $h_{\varepsilon_{t}}$ \\
\hline Axial & 163.35 & -85.1 & 275.8 & 38.64 & -93.1 & 122.3 & 345 & 17.34 \\
\hline Shear & 122.63 & -120.91 & 101.61 & 1027 & 37051 & 131.12 & 109.27 & 12.4 \\
\hline
\end{tabular}




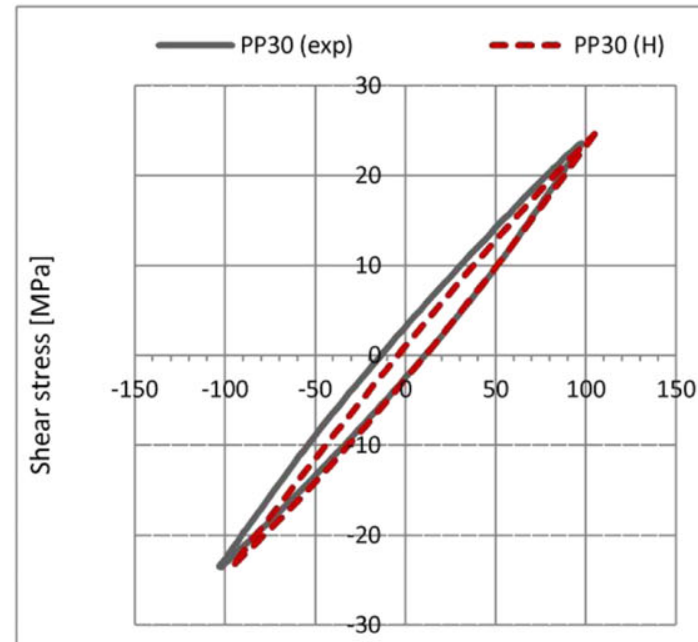

(A)

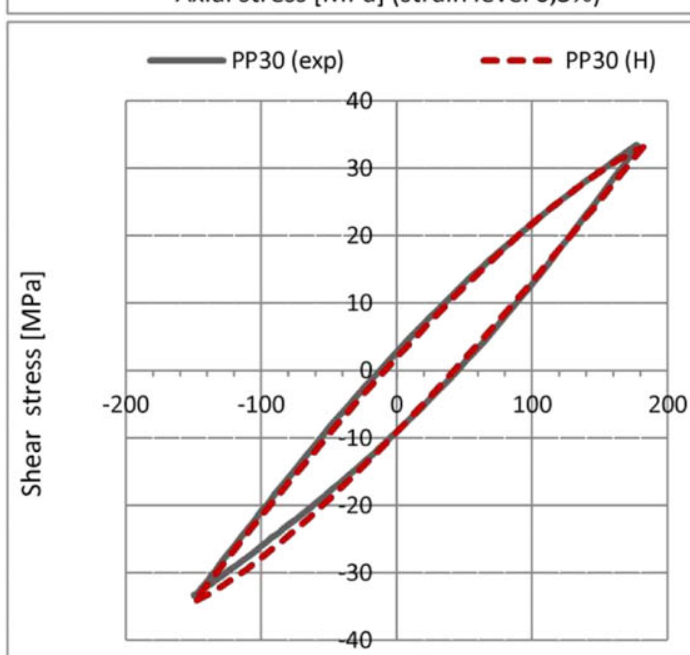

(C)

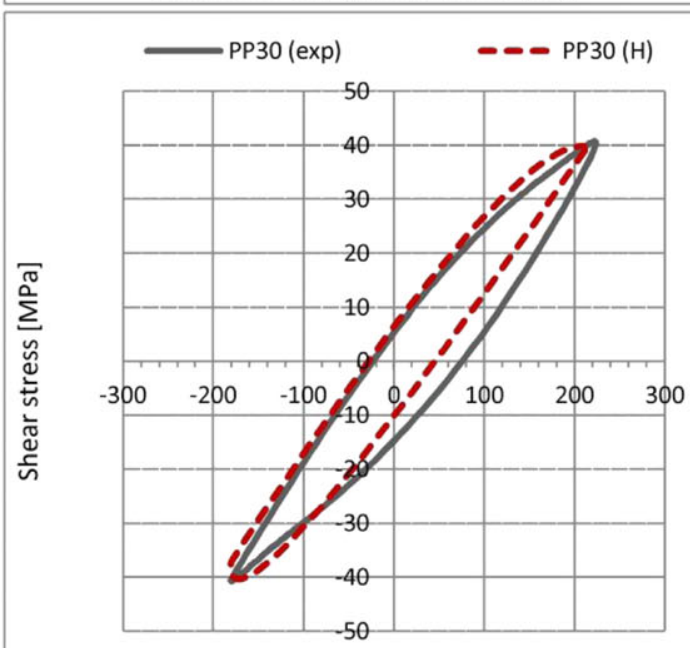

(E) Axial stress [MPa] (strain level 1\%)

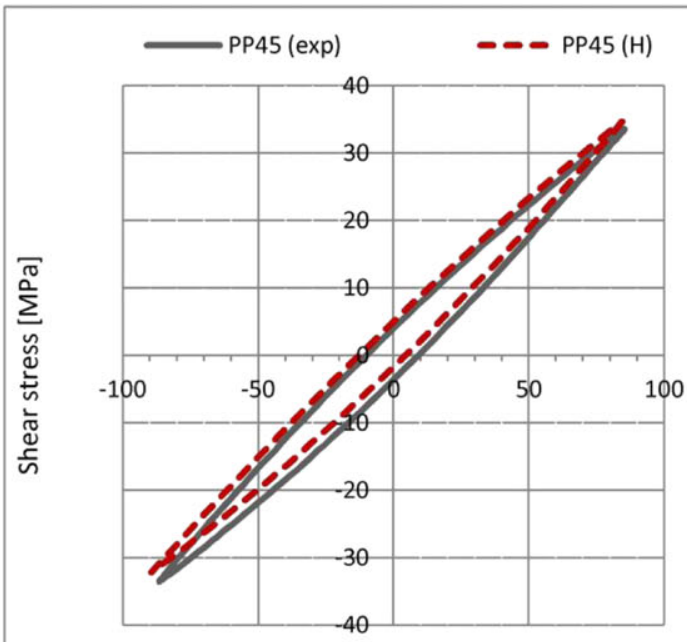

(B) Axial stress [MPa] (strain level 0,3\%)

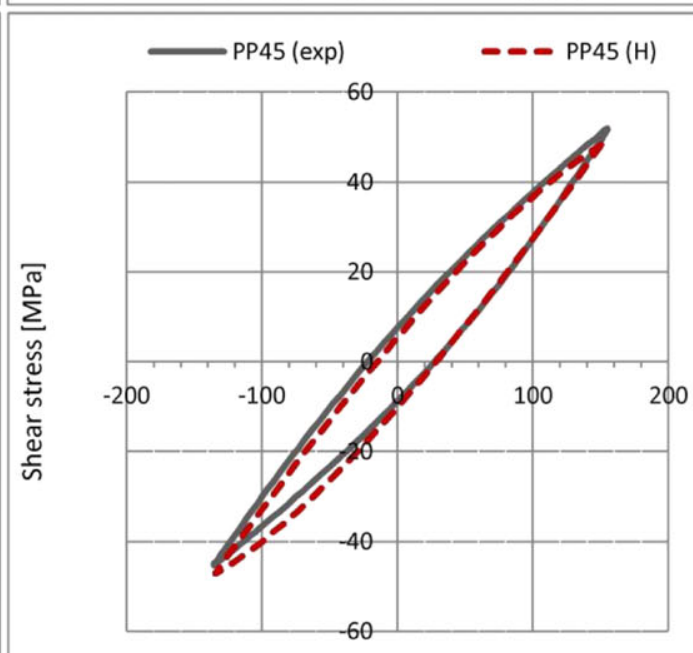

(D) Axial stress [MPa] (strain level 0,6\%)

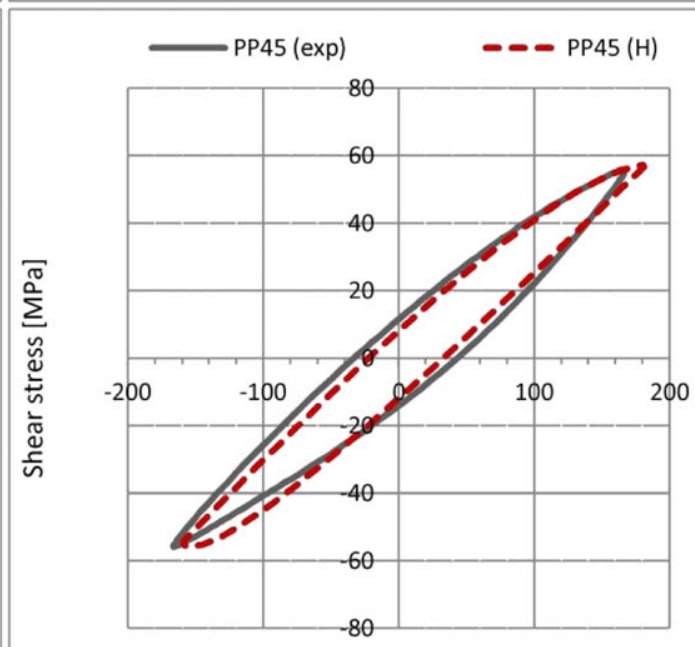

(F) Axial stress [MPa] (strain level 1\%)

FIGURE 15 Correlation between estimations and experiments for cases: A,C,E, Case 3, and B,D,F, Case 4 (exp-experiments; Hphenomenological model) [Colour figure can be viewed at wileyonlinelibrary.com] 


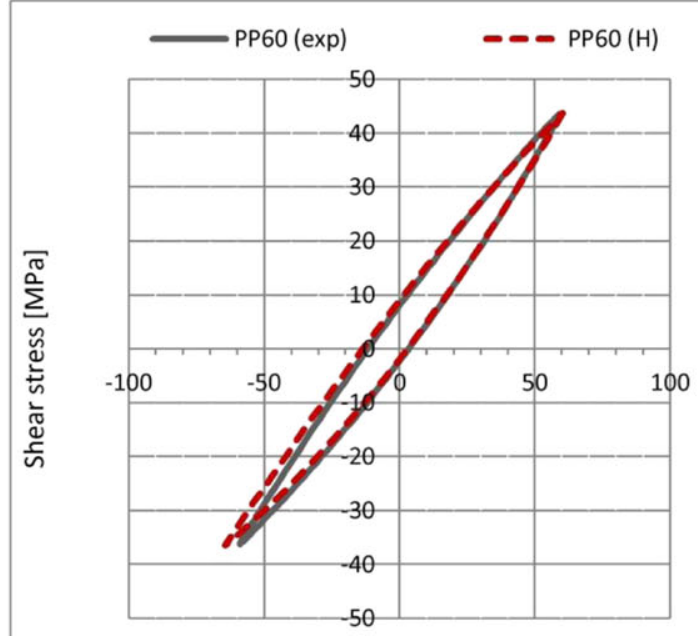

(A)

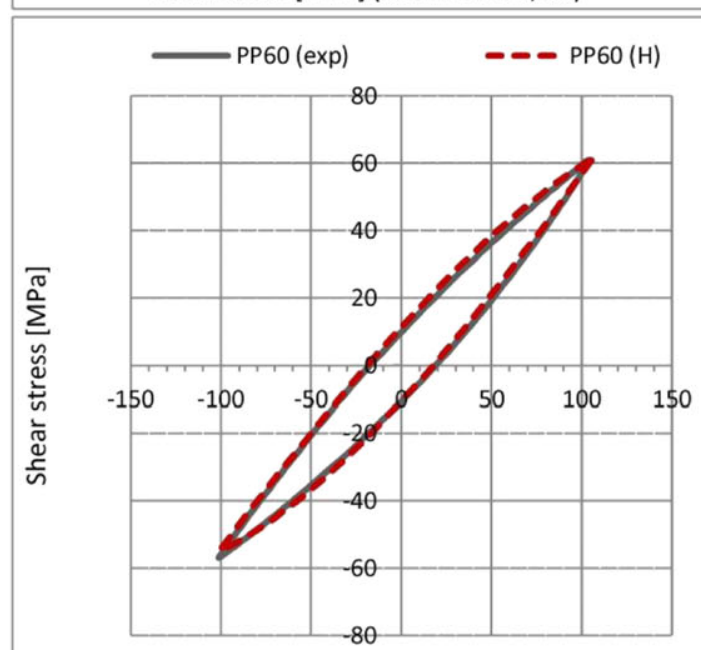

(C)

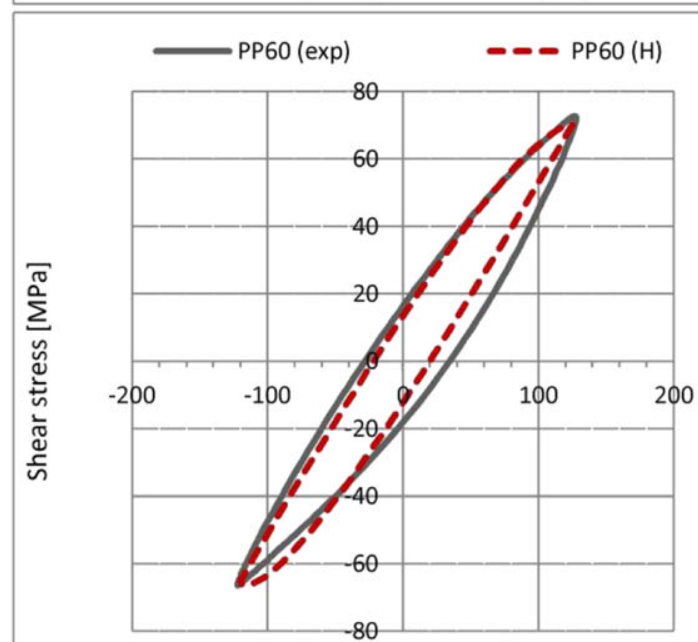

(E) Axial stress [MPa] (strain level 1\%)

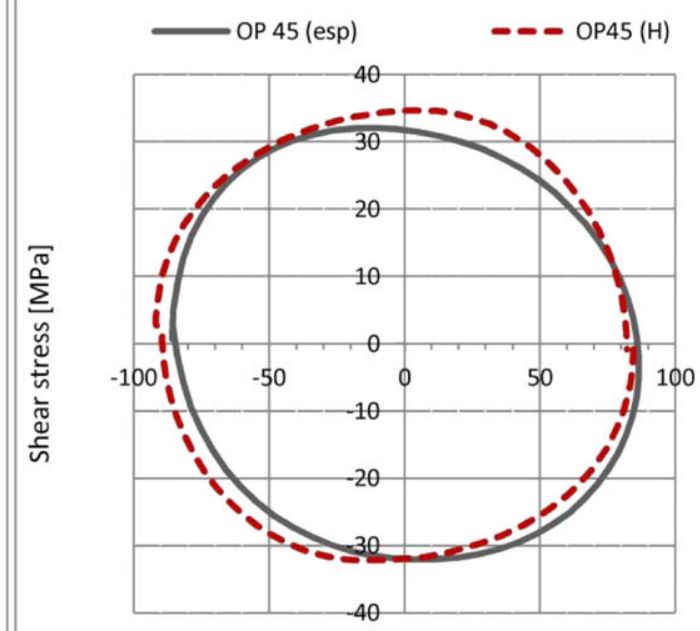

(B) Axial stress [MPa] (strain level 0,3\%)

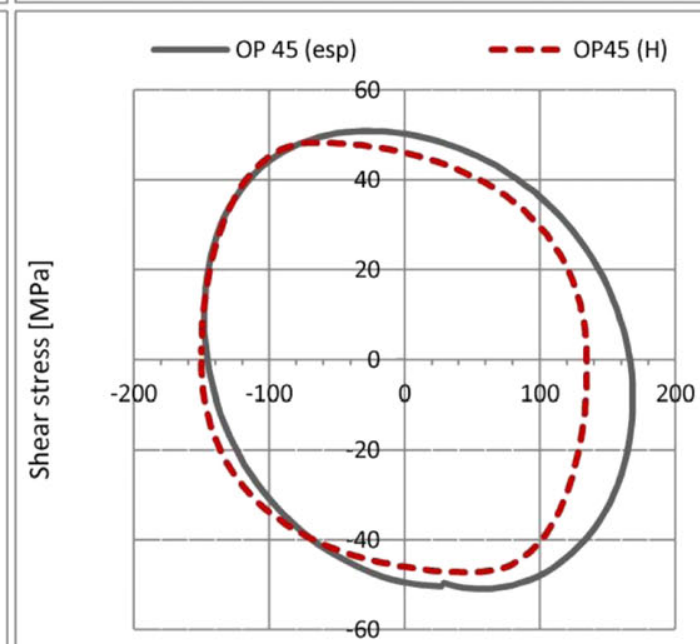

(D) Axial stress [MPa] (strain level 0,6\%)

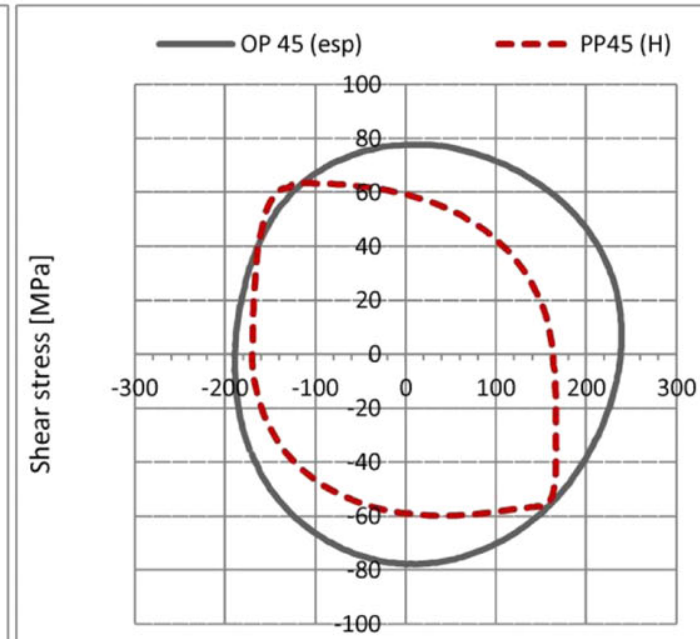

(F) Axial stress [MPa] (strain level 1.14\%)

FIGURE 16 Correlation between estimations and experiments for cases: A,C,E, Case 5, and B,D,F, Case 6 (exp-experiments; Hphenomenological model) [Colour figure can be viewed at wileyonlinelibrary.com] 
the axial component that it can be found the greatest differences, especially in points $\mathrm{P} 3, \mathrm{P} 4, \mathrm{P} 5$, and P6.

Therefore, from Figure 14, it can be concluded that the phase shift effect in the cyclic deformation of the AZ31B$\mathrm{F}$ magnesium alloy only influences the axial cyclic response under OP45 loading paths.

The AZ31B-F curves shown in Figures 6 to 9 were used to obtain the polynomials constants presented in Equations (7) to (8). Tables 1 and 2 show these constants for the proportional loadings considered in the phenomenological approach, ie, cases 1 to 5 .

As example, Table 3 shows the results obtained to estimate the hysteresis loops of loading Case 3 (SAR, $\lambda=30^{\circ}$ ) at $0.6 \%$ of strain level $\left(\varepsilon_{s l}\right)$. In rows 2 and 3 is shown the $\mathrm{P}$ point values for this case, which are used to obtain the third-degree polynomials presented in rows 5 and 7 .

Figures 15 and 16 show the correlation between the developed phenomenological model, represented by the letter $\mathrm{H}$, and experiments. It was selected three values for strain level in order to compare the results under three levels of cyclic plasticity.

To make this analysis, it is presented the results in stress space, where the axial stress vs shear stress is presented. Here, neither the shear stress nor the axial stress results are affected by any factor as seen in the von Mises stress space, for instance.

In the testing machine console, the strain-time variation strictly follows the loading paths depicted in Figure 3; thus, it would be expected that the inherent stress variations should have the same loading path pattern. However, from the AZ31B-F experiments, it can be concluded that the results are quite different from the expected ones; much of this difference is related with the magnesium cyclic plastic behaviour.

From the results presented in Figures 15 and 16, it can be concluded that the developed model follows well the proportional loading paths obtained by experiments; only in Cases 3 and 5 at 1\% of strain level it was found a slight deviation from the experimental results, see Figures 15E and $16 \mathrm{E}$.

The advantage of the developed phenomenological model comparatively to constitutive models is the possibility to simulate the effect of the SAR in the material's elastoplastic cyclic behaviour and consider other plastic effects such as twinning and de-twinning effects, for instance. These effects became more relevant under higher strain level values. Figure 16A,C,E presents the results for Case 5, which have a SAR equal to $60^{\circ}$; in this case, the shear strain is higher than the axial one. At $1 \%$ of total strain level, the developed model has a slight deviation in Case 3.

The nonproportional estimates displayed in Figures 15 and 16 were made considering the proportional estimates of Case $4\left(\operatorname{SAR} 45^{\circ}\right)$ and adding a $90^{\circ}$ phase angle to the axial and shear cyclic responses of Case 4 . This approach fails to follow the experimental results, only for a strain level equal to $0.3 \%$; the results were acceptable as seen in Figure 16B, where the estimates almost follow the experimental results. For $0.6 \%$ of total strain, the differences between estimates and experiments start to increase, where nonproportional hardening starts to be more pronounced.

For $1.14 \%$ of total strain level, see Figure 16F, the developed model estimates are inside of the experimental loading path; in this case, the stress values are lower than they should be indicating that the developed model does not capture the magnesium's nonproportional hardening; this evidence becomes more obvious with the increment of the total strain level value. Based on these results, it can conclude that the approach to estimate nonproportional cyclic behaviour based on proportional loadings, considering the same SAR in both proportional and nonproportional loadings, can be fairly considered for total strain level lower than $0.3 \%$.

\section{5 | CONCLUSIONS}

In this work, it was presented a phenomenological approach to model the cyclic behaviour of anisotropic materials under uniaxial and multiaxial loading conditions. The approach was applied to model the cyclic behaviour of the AZ31B-F magnesium alloy where an experimental program comprising several proportional loading paths and one nonproportional loading path was carried out. Based on these experiments, the AZ31B-F cyclic behaviour was mapped by polynomial functions where the relation between biaxial stresses, total strains, plastic strains, and back stresses was modulated. The SAR effect in the elastic-plastic parameters under proportional and nonproportional loadings was analysed; also, a comparison between proportional and nonproportional loadings with the same SAR was analysed to infer about the effect of phase shift in the AZ31B-F cyclic behaviour. Results show that the experimental hysteresis loops are also asymmetric under multiaxial loading conditions, especially the ones from the axial loading components; the asymmetry found in the shear loading components came from the first loading direction. The developed model shows limitations that need to be overcome, ie, the lack of a parameter that tunes nonproportional hardening for different SARs. Despite this limitation, the developed model shows good agreement with the experimental data under uniaxial and proportional loadings, and the model captures very 
well the plastic strains and back stresses for a wide range of loading conditions.

\section{ACKNOWLEDGEMENTS}

The authors gratefully acknowledge financial support from Fundação para Ciência e Tecnologia, through the project PTDC/EME-PME/104404/2008 and Ph.D. Grant $\mathrm{PD} / \mathrm{BD} / 52344 / 2013$.

\section{ORCID}

Vitor Anes (D) https://orcid.org/0000-0002-8526-398X

Luís Reis iD https://orcid.org/0000-0001-9848-9569

\section{REFERENCES}

1. Jiang Y, Zhang J. Benchmark experiments and characteristic cyclic plasticity deformation. Int J Plast. 2008;24(9):1481-1515.

2. Mousa JAB. Multiaxial fatigue characterization and modeling of AZ31B magnesium extrusion. 2011.

3. Wang H, Wu PD, Wang J, Tomé CN. A crystal plasticity model for hexagonal close packed (HCP) crystals including twinning and de-twinning mechanisms. Int J Plast. 2013;49:36-52.

4. Bruni C, Forcellese A, Gabrielli F, Simoncini M. Post-welding formability of AZ31 magnesium alloy. Mater Des. 2011;32(5): 2988-2991.

5. Albinmousa J, Jahed H, Lambert S. An energy-based fatigue model for wrought magnesium alloy under multiaxial load. In: ICMFF9. ; 2013.

6. Albinmousa J, Jahed H, Lambert S. Cyclic axial and cyclic torsional behaviour of extruded AZ31B magnesium alloy. Int $J$ Fatigue. 2011;33(11):1403-1416.

7. Reis L, Anes V, de Freitas M. AZ31 magnesium alloy multiaxial LCF behavior: theory, simulation and experiments. Adv Mat Res. 2014;891:1366-1371.

8. Jiang Y, Kurath P. A theoretical evaluation of plasticity hardening algorithms for nonproportional loadings. Acta Mech. 1996;118(1-4):213-234.
9. Koike J. Enhanced deformation mechanisms by anisotropic plasticity in polycrystalline $\mathrm{Mg}$ alloys at room temperature. Metall Mater Trans A. 2005;36(7):1689-1696.

10. Wang H, Raeisinia B, Wu PD, Agnew SR, Tomé CN. Evaluation of self-consistent polycrystal plasticity models for magnesium alloy AZ31B sheet. Int J Solids Struct. 2010;47(21):2905-2917.

11. Wang H, Wu PD, Wang J. Modeling inelastic behavior of magnesium alloys during cyclic loading-unloading. Int $J$ Plast. 2013;47:49-64.

12. Wu L, Jain A, Brown DW, et al. Twinning-detwinning behavior during the strain-controlled low-cycle fatigue testing of a wrought magnesium alloy, ZK60A. Acta Mater. 2008;56(4): 688-695.

13. Chung K, Lee M-G, Kim D, Kim C, Wenner ML, Barlat F. Spring-back evaluation of automotive sheets based on isotropic-kinematic hardening laws and non-quadratic anisotropic yield functions: part I: theory and formulation. International Journal of Plasticity. 2005;21:861-882.

14. Reis LG, Anes V, Li B, de Freitas M. Characterizing the cyclic behaviour of extruded AZ31 magnesium alloy. In: Materials Science Forum. Vol 730. Zurich, Switzerland: Trans Tech Publ; 2013:727-732.

15. Anes V, Reis L, Li B, Fonte M, de Freitas M. New approach for analysis of complex multiaxial loading paths. International Journal of Fatigue. 2014;62:21-33.

16. Anes V, Reis L, Freitas M. Evaluation of the AZ31 cyclic elasticplastic behaviour under multiaxial loading conditions. Fract Struct Integrity. 2014;8(30):282-292.

17. Madrigal C, Chaves V, Navarro A. Numerical implementation of a multiaxial cyclic plasticity model for the local strain method in low cycle fatigue. Fract Struct Integrity. 2014;8(30):153-161.

How to cite this article: Anes V, Reis L, de Freitas M. Evaluation of a phenomenological elastic-plastic approach for magnesium alloys under multiaxial loading conditions. Fatigue Fract Eng Mater Struct. 2019;42:2468 -2486. https://doi.org/ $10.1111 /$ ffe. 13025 